\title{
1 Capturing in situ Virus-Host Range and Interaction Dynamics through Gene Fusion with
}

\section{2 epicPCR}

3 Eric G. Sakowski ${ }^{*}$, Keith Arora-Williams ${ }^{1}$, Funing Tian ${ }^{2}$, Ahmed A Zayed $^{2}$, Olivier Zablocki ${ }^{2}$,

4 Matthew B. Sullivan ${ }^{2,3}$, Sarah P. Preheim ${ }^{1 *}$

$6{ }^{1}$ Department of Environmental Health and Engineering, Johns Hopkins University, MD, USA

72 Department of Microbiology, The Ohio State University, OH, USA

83 Department of Civil, Environmental and Geodetic Engineering, The Ohio State University, $\mathrm{OH}$

9 USA

$11 *$ Corresponding authors

13 Abstract

14 Viruses impact microbial diversity, phenotype, and gene flow through virus-host

15 interactions that in turn alter ecology and biogeochemistry. Though metagenomics surveys are

16 rapidly cataloging viral diversity, capturing specific virus-host interactions in situ would identify

17 hosts for novel viruses and reveal influential ecological or environmental factors. We leveraged

18 metagenomics and a high-throughput, cultivation-independent gene fusion technique (epicPCR)

19 to investigate viral diversity and virus-host interactions over time in a critical estuarine

20 environment, the Chesapeake Bay. EpicPCR captured in situ virus-host interactions for viral

21 clades with no closely related database representatives. Abundant freshwater Actinobacteria

22 lineages were the most common hosts for these poorly characterized viruses, and observed viral

23 interactions with one abundant Actinobacterial population (Rhodoluna) were correlated with 
24 environmental factors. Tracking virus-host interaction dynamics also revealed ecological

25 differences between multi-host (generalist) and single-host (specialist) viruses. Generalist viruses

26 had significantly longer periods with observed virus-host interactions but specialist viruses were

27 observed interacting with hosts at lower minimum abundances, suggesting more efficient

28 interactions. Together, these observations reveal ecological differences between generalist and

29 specialist viruses that provide insight into evolutionary trade-offs. Capturing in situ interactions

30 with epicPCR revealed environmental and ecological factors that shape virus-host interactions,

31 highlighting epicPCR as a scalable new tool in viral ecology. 


\section{Introduction}

Viruses impact the diversity and function of microbial communities from the open ocean ${ }^{1}$

35 and soils ${ }^{2}$ to the human gut ${ }^{3}$. Bulk measurements implicate viruses as major contributors to daily

36 bacterial mortality $\left(20-40 \%\right.$ in surface waters $\left.{ }^{4}\right)$, carbon export ${ }^{5}$, and biogeochemical cycling ${ }^{6}$. In

37 the Chesapeake Bay, the largest estuary in the United States, high viral production rates $(\sim 8 \mathrm{x}$

$3810^{6}$ viruses $\mathrm{ml}^{-1} \mathrm{~h}^{-1}$ ) suggest up to $20 \%$ of the bacterial community can be lysed by viruses per

39 hour, $^{7}$ although environmental factors, such as tidal mixing of fresh and marine water, could

40 modulate viral production and viral-mediated mortality, as observed in other ecosystems ${ }^{8}$. These

41 community-level impacts are the aggregate of a myriad of individual virus-host infections that

42 result in an estimated $10^{23}$ infections per second in the oceans worldwide ${ }^{9}$. However,

43 cultivation ${ }^{10,11}$ and theoretical models ${ }^{12,13}$ suggest viral pressure is not equally distributed across

44 the microbial community, implying a subset of virus-host infections could contribute

45 disproportionately to microbial mortality with implications for microbial community diversity

46 and biogeochemical cycling. Since individual virus-host interactions (i.e. any physical

47 association that could lead to an active infection including attachment, injection of genetic

48 material, latent period, lysogeny) could be influenced by different ecological and environmental

49 factors, establishing connections between virus-host interactions and environmental factors will

50 be the first step in fine-tuning ecosystem models to better predict variability in viral productivity

51 and bacterial mortality.

52 Viral metagenomics has greatly expanded our knowledge of environmental viral

53 diversity; yet, linking these viruses to their hosts remains a bottleneck for investigating the

54 relative ecosystem impact of individual virus-host pairs. Bioinformatics approaches applied to

55 bacterial and viral shotgun metagenomes show promise for identifying hosts for viral 
communities. A recent study linked $35 \%$ of shotgun-assembled soil viral populations to putative

57 hosts through a combination of bioinformatics approaches for host identification, such as

58 CRISPR spacer matches, sequence homology, and k-mer frequencies ${ }^{2}$. Recent floods of oceanic

59 virus data ${ }^{14}$ and metagenome-assembled genomes (MAGs) ${ }^{15}$ will likely improve in silico host

60 predictions for marine viruses. Still, it is estimated that only $10 \%$ of uncultivated bacterial

61 lineages contain CRISPR-Cas systems ${ }^{16}$, limiting efforts to link viruses and hosts by CRISPR

62 spacer homology. Comparisons of mycobacteriophage indicate some viruses may switch hosts

63 too quickly for their genomes to evolve similar DNA signatures to their host ${ }^{17}$, thus constraining

64 in silico predictions via k-mer frequencies. Other techniques, like spot, plaque and liquid assays ${ }^{18}$

65 and viral tagging ${ }^{19,20}$ require cultivation of the host, preventing investigations of viral

66 interactions for the majority of bacterial populations that cannot be readily cultivated.

67 Cultivation-independent approaches like the microscopy-based phageFISH ${ }^{21}$ and microfluidic

68 digital polymerase chain reaction $(\mathrm{PCR})^{22}$ either require laborious and database-dependent probe

69 design and optimization or fail to scale due to few micro-chambers yielding positive interactions,

70 respectively. Furthermore, these methods cannot identify virus-host interaction dynamics or the

71 ecological and environmental factors that influence these interactions. Thus, there is a need for

72 new, scalable experimental methods that capture virus-host interactions in situ.

73 Here we sought to investigate the ecology of viruses interacting with key bacterial

74 populations in the Chesapeake Bay. We leveraged viral metagenomics to study viral diversity

75 and seasonal dynamics at population-scale resolution ${ }^{19}$. Furthermore, we repurposed emulsion

76 paired isolation-concatenation PCR (epicPCR) ${ }^{23}$ to evaluate in situ virus-host interaction

77 dynamics of ecologically important bacterial populations. This analysis revealed that abundant

78 freshwater Actinobacteria populations were the primary host of poorly characterized viruses 
sharing genetic similarity with cyanosipho-and-podoviruses. Additionally, environmental factors partially explained the observed interactions between one abundant Actinobacterial population

81 (Rhodoluna) and viral clades. We also identified ecological differences (i.e. observed

82 "interaction life-span" and minimum host abundance) between specialist and generalist viruses.

83 These results demonstrate that this approach can be applied as a scalable and complementary

84 new tool in viral ecology.

\section{Results}

\section{Chesapeake Bay viral populations are novel, endemic and seasonally dynamic}

To investigate temporal changes in viral populations and virus-host interactions, water samples were collected from May 2017 and May to December 2018 in the Rhode River, a tidal estuary on the Western Shore of the Chesapeake Bay (Fig. S1). We employed short-read shotgun

91 metagenomics to the bacterial fraction and short-/long-read hybrid metagenomic approaches ${ }^{24}$ to

92 the viral fraction of samples to investigate bacterial and viral diversity, respectively. In total,

939,392 viral populations (approximately species level genotypes dereplicated using 95\% ANI over

$9480 \%$ of the contig; size $>5 \mathrm{~kb}^{14,25-27}$ ) were assembled from the metagenomic libraries $-7,801$

95 from the viral fraction and 1,591 from microbial-fraction $(>0.2 \mu \mathrm{m})$ metagenomes. Viral

96 diversity in the Chesapeake Bay was distinct from reference database representatives.

97 Chesapeake Bay viral populations formed 473 clusters in a network analysis of shared genes ${ }^{28}$,

98 only $40(8 \%)$ of which also contained RefSeq representatives. Over 2,000 additional Chesapeake

99 Bay viral populations were not assigned a single cluster. Chesapeake Bay viral populations also

100 shared little overlap with the Global Ocean Virome 2.0 (GOV 2.0) dataset $^{14}$ (approximately $1 \%$

101 of the viral populations could be detected in any GOV2.0 sample) and other estuarine samples ${ }^{29-}$ 
even after accounting for differences in sequencing depth between samples (Fig. 1). This

103 highlights the novelty and potentially endemic nature of Chesapeake Bay viral populations and is

104 consistent with prior inferences of endemism from cyanophage gene markers ${ }^{32}$. This endemism

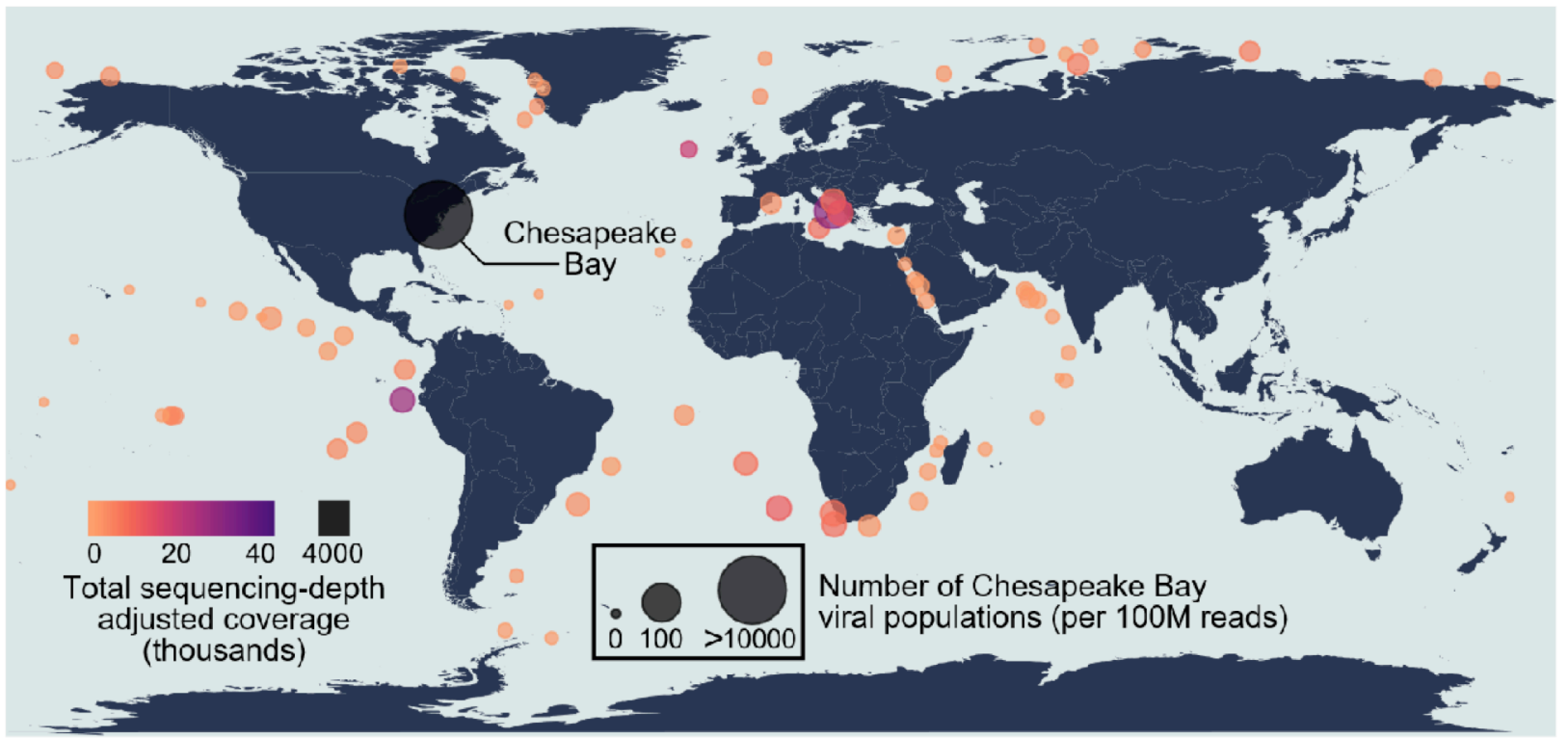

Figure 1. Global distribution and abundance of Chesapeake Bay viral populations. The Chesapeake Bay viral populations were highly endemic as less than 100 populations were shared with any other location in the global ocean viromes (GOV2.0) dataset (circle size) and recruited significantly less reads from the GOV2.0 stations (circle color). A viral population was considered detected in a GOV2.0 sample if the sample's reads covered at least $70 \%$ of the genomic length of the viral population. Due to differences in the sequencing depth between libraries, the number of shared viral populations and their total coverage (tpmean; see Materials and Methods) were adjusted for each library size. For the stations that have multiple samples, the maximum value is reported.

Consistent with previous reports from the Chesapeake Bay ${ }^{34,35}$, bacterial and viral

109 shared nearly three times more viral populations with a winter 2012 sample $^{36}$ than with spring 110 and summer samples from the same year (i.e. 2018; Figs. 2B, S2). Likewise, spring 2017 and 
1112018 samples shared half of their viral populations with each other but fewer than $30 \%$ of their

112 viral populations with other seasons in 2018 (Figs. 2B, S2), illustrating that Chesapeake Bay

113 viral communities had greater seasonal than inter-annual variability. The number of shared

114 populations between samples was similar whether comparing across entire viral contigs or a

115 single gene. Ribonucleotide reductase (RNR) genes, which are broadly distributed across aquatic

116 dsDNA lytic viruses ${ }^{37}$, were extracted from the $>5 \mathrm{~kb}$ viral contigs and clustered at $95 \%$

117 nucleotide identity. The number of shared viral populations between samples derived from this

118 single gene was highly correlated with the number of shared populations using entire contigs $\left(\mathrm{r}^{2}\right.$

$119=0.95, \mathrm{p}<0.001$; Fig. S3), marking RNRs as a good proxy of aquatic viral diversity in our

120 study.

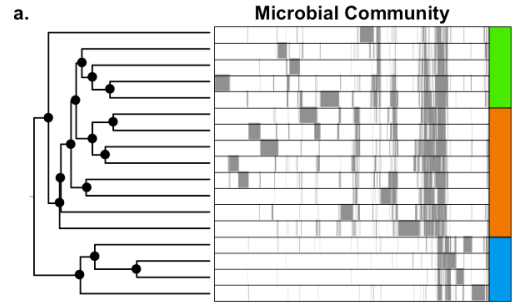

Jackknife Support: • $100 \% \quad \circ 56 \%$

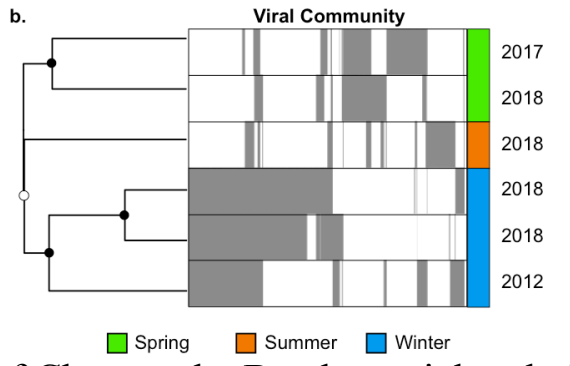

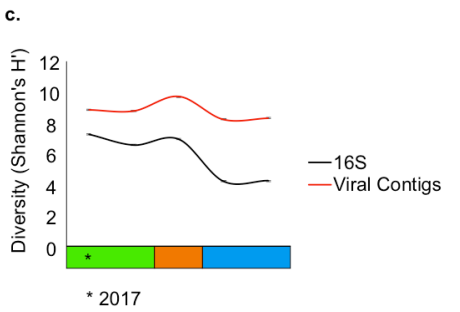

2017

Figure 2. Seasonal dynamics of Chesapeake Bay bacterial and viral communities. A) Hierarchical clustering of bacterial communities based on 16S rRNA gene sequences shows that microbial communities are most similar within the same season. Libraries were subsampled at 150,000 observations with ten jackknifed replicates. Individual sequence variants are colored gray. B) Hierarchical clustering of viral communities from viral populations $>5 \mathrm{~kb}$ collected across different years (see Materials and Methods) shows that seasonal community similarity exceeds within-year community similarity. Libraries were sub-sampled at 300,000 observations. Individual viral populations are colored gray. For the deeply-sequenced 2012 sample, only viral populations that were observed in the shallower 2017-2018 samples were included for clarity. C) Bacterial and viral diversity (Shannon's $H^{\prime}$ index) for paired 16S rRNA gene and viral metagenomes were significantly correlated $\left(\mathrm{r}^{2}=0.6, \mathrm{p}<0.05\right)$. Viral metagenomes were subsampled to the smallest metagenome $(15$ M reads) prior to assembly for all Shannon's $H^{\prime}$ diversity analyses. Diversity indices represent an average of ten jackknifed replicates at a sampling depth of 10,000 observations. Error bars are SD. 
124 bioinformatics and experimental approaches. First, we inferred hosts associated with observed

125 viral RNR genes through similarity to reference sequences with known bacterial hosts. RNR

126 genes observed in the spring most frequently shared homology with myoviruses that infect

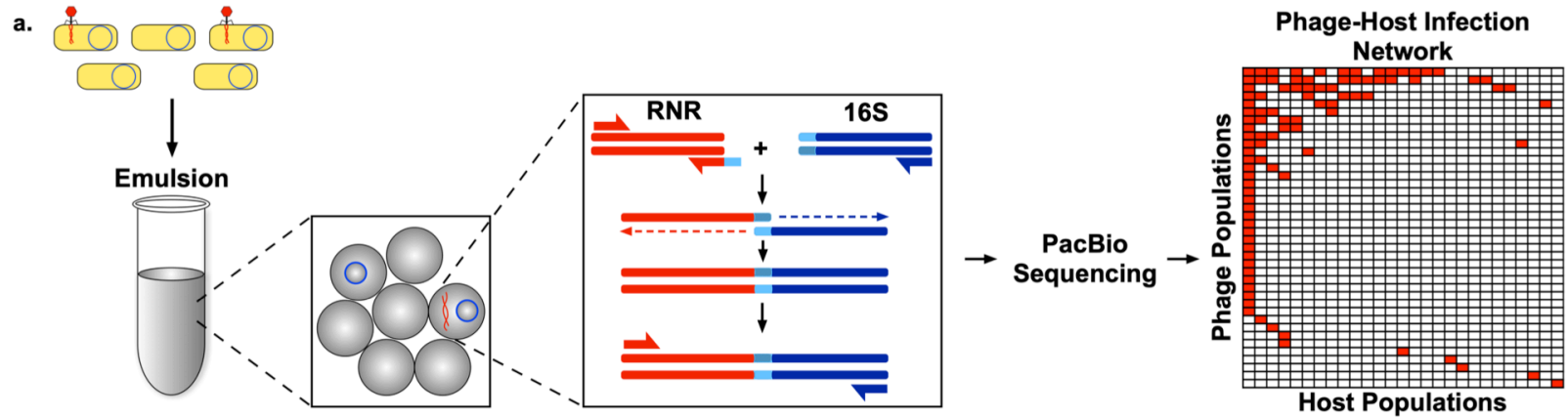

b.

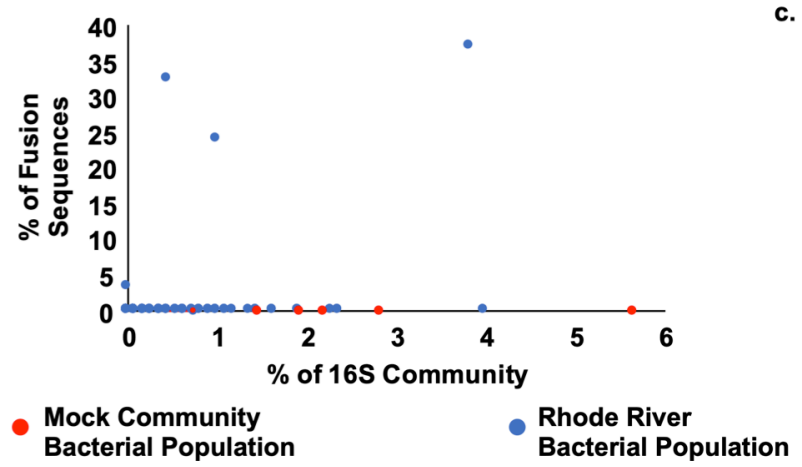

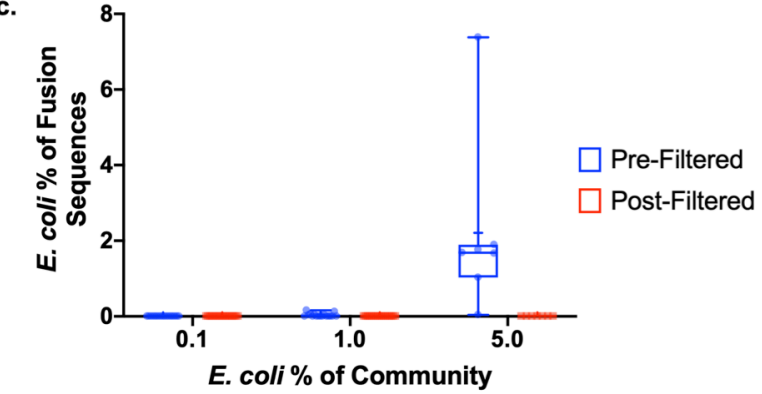

Fig. 3. EpicPCR identifies phage-host interactions in the environment without cultivation. A) Overview of the experimental design for epicPCR, identifying phage-host interactions through single-cell isolation in emulsion droplets and virus and host marker gene fusion. Left: individual cells are isolated within emulsion droplets and the genome of the host (blue circle) and virus (red curved lines) serve as the template for the gene fusion reaction. Middle: fusion PCR joins and amplifies viral and host marker genes from actively infected cells within emulsion droplets. RNR (ribonucleotide reductase; red) and 16S (16S rRNA gene; blue) are joined through an overlapping primer sequence (light blue). Right: fused amplicons are sequenced and analyzed to identify the network of viral-host interactions in the environment. Overall, 95 interactions (red cells) between 40 unique phage RNR sequences and 27 unique host $16 \mathrm{~S}$ rRNA sequences were identified. B-C) Specificity of the method was tested by spiking Rhode River water samples with a mock community or single uninfected host (E. coli) prior to emulsification. B) Proportion of fusion sequences belonging to uninfected mock (red) and Rhode River (blue) community members as a function of their community abundance. Uninfected mock community sequences were not found to be associated within any fusion products. C) Proportion of fusion products containing E. coli when spiked into Rhode River samples at $0.1 \%(\mathrm{n}=17), 1 \%(\mathrm{n}=17)$, and $5 \%(\mathrm{n}=7)$ of the community. Post-filtered sequences are those interactions observed in a minimum of three libraries. 
127 Cyanobacteria and Gammaproteobacteria, while winter samples showed an increase in

128 podoviruses infecting Alphaproteobacteria. August samples were largely composed of

129 populations with no viral reference database representatives (Fig. S4a), and overall $54 \pm 19 \%$ of

130 the RNR sequences were not classified using this homology-based approach. However, even

131 'classifiable’ RNR sequences were often only distantly related to viral reference sequences (Fig.

132 S4b), and homology to viral references does not necessarily indicate a common host. For

133 example, Pelagibacter phage HTVC008M and T4-like cyanophages share $71 \%$ RNR nucleotide

134 identity and would be classified together by our cutoffs; yet, they infect hosts from different

135 phyla. Therefore, gene homology may aid predictions of phage morphology but lacks the

136 resolution to predict hosts for environmental viruses that are only distantly related to reference

137 database representatives.

To further identify hosts associated with viral RNR sequences, we modified an existing

139 single-cell gene fusion protocol (epicPCR) $)^{23}$ and applied it to a large set of samples from our

140 study site (Fig. S1b). EpicPCR takes advantage of the close physical proximity of phage and host

141 DNA within infected bacterial cells to fuse viral marker and host 16S ribosomal RNA (rRNA)

142 genes within emulsion droplets, providing single-cell level resolution (Fig. 3). The method

143 requires no a priori knowledge of putative hosts. However, because viruses lack a universal

144 marker gene ${ }^{38}$, viral diversity assessed by epicPCR is limited by viral marker gene choice. Based

145 on reference genomes, cyanosipho-and-podoviral RNR genes form a distinct, monophyletic

146 clade (the Cyano SP clade ${ }^{39}$ ), which enabled the development of primers to target these viral

147 populations by epicPCR. Furthermore, cyanopodoviruses were shown to be the dominant

148 cyanophage populations in the Chesapeake Bay in previous studies ${ }^{34}$; yet, cyanosipho-and-

149 podoviral RNR genes from the Chesapeake Bay viral metagenomes were only distantly related to 
150 reference cyanophage sequences (Fig. S4b). Therefore, Cyano SP RNR was an ideal marker gene

151 choice for investigating poorly characterized cyanophage-like diversity in this system.

152 We validated the specificity of epicPCR using uninfected mock communities and spike-

153 in controls. With a complex mock community spiked into an environmental sample, uninfected

154 control cells comprised as much as $16 \%$ of the total community and accounted for up to four of

155 the ten most abundant community members. Despite their dominance, mock community control

156 sequences were not observed in any fusion products (Fig. 3B) Additionally, we ensured

157 specificity by adding uninfected $E$. coli cells to replicate epicPCR reactions from samples

158 collected between May and December 2018, to control for non-specific interactions. Although

159 uninfected control sequences were occasionally observed in the fusion data, these non-specific

160 interactions were not consistently associated with any specific phage sequence. Thus, non-

161 specific associations were removed by requiring that virus-host sequence pairs be observed in at

162 least three libraries to be considered a positive interaction (Fig 3C).

164 identity across at least three samples (out of 58 total samples). The fusion amplicons contained

16527 unique hosts (16S rRNA gene sequence at 100\% identity), 40 unique phages (RNR at 100\%

166 identity), and 95 unique phage-host interactions between May and December 2018 (Fig. 4). The

167 RNR genes identified from the epicPCR experiment formed three distinct phylogenetic clades

168 (Fig. 4A) and shared greatest homology with T7-like Chesapeake Bay cyanopodoviral isolates S-

$169 \mathrm{CBP} 1, \mathrm{~S}-\mathrm{CBP} 3$, and S-CBP4 but had less than $80 \%$ nucleotide identity to any reference sequence

170 (Table S1). Recruiting viral metagenome reads to the RNR amplicons demonstrated these viral

171 RNR sequences were not very abundant in our samples and highlights the sensitivity of the

172 epicPCR method (Fig. S5). These RNR sequences failed to assemble into longer contigs, likely 
173 due to their low abundances and micro-diversity, which can prevent the assembly of even

174 abundant phage genomes ${ }^{40}$.

a.

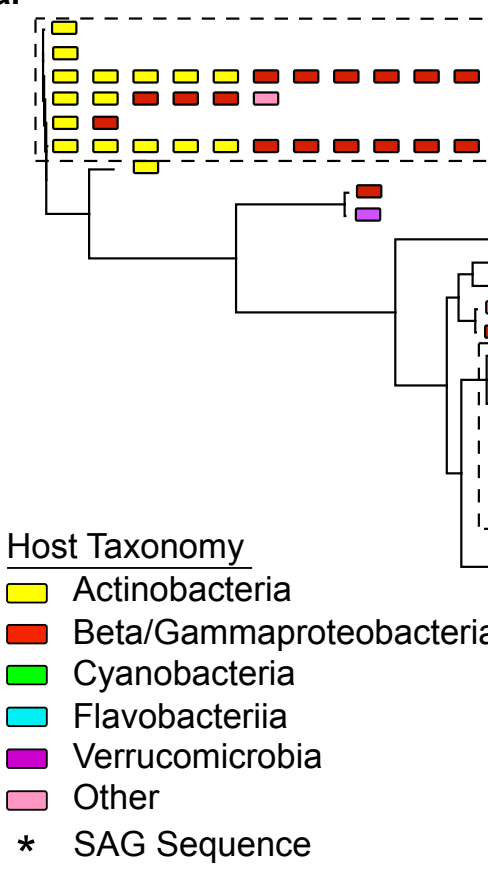

0.3 b.

Clade I

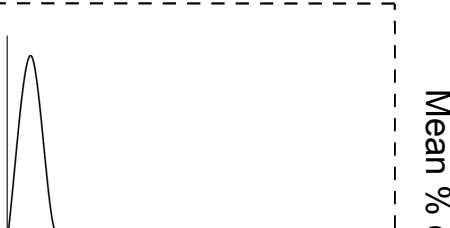

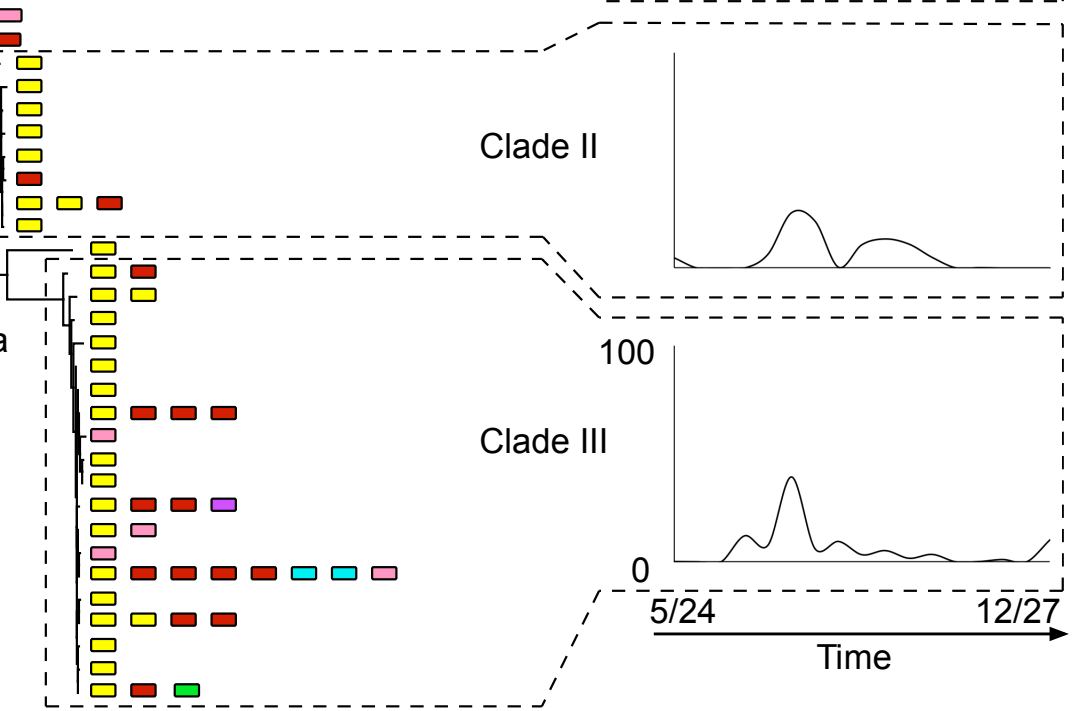

c.
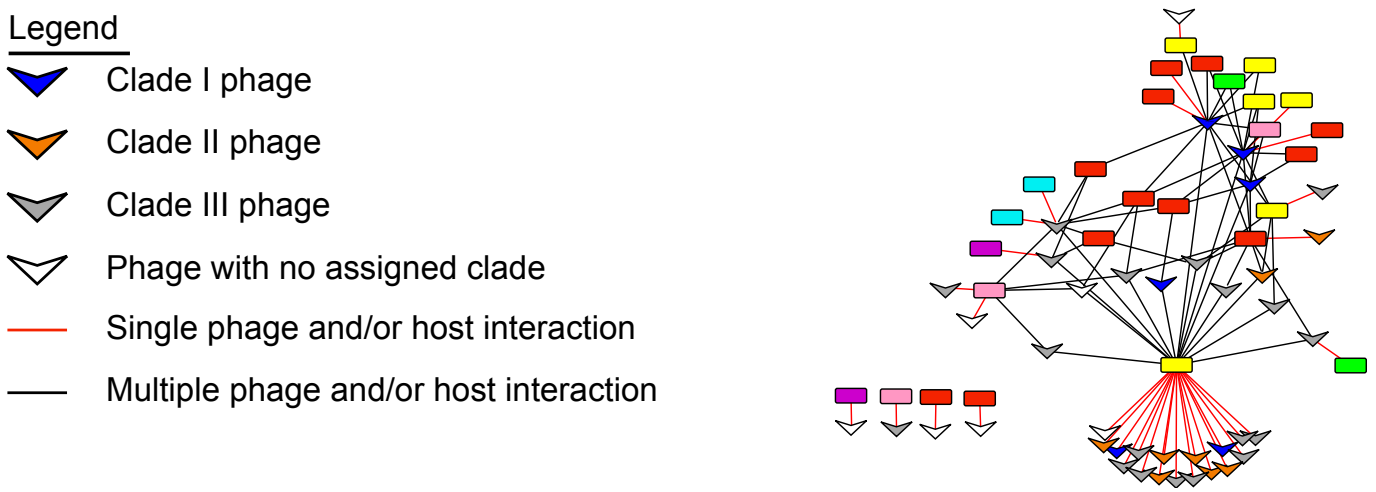

Figure 4. Abundance, diversity, and ecology of 'Cyano SP'-like phage-host interactions. A) Maximum likelihood tree with 100 bootstrap replicates of Chesapeake Bay 'Cyano SP'-like phage RNR gene sequences (G1380 to A2079 in E. coli $n r d A$ ). Only RNR gene sequences that were observed with the same host (100\% nucleotide identity of 16S rRNA gene) in at least three epicPCR fusion amplicon libraries were included. Phage RNR sequences were clustered at $100 \%$ nucleotide identity and host representatives were plotted on the tree. Host taxonomy identified by 16S rRNA gene homology for each phage RNR gene sequence is indicated. Scale bar represents nucleotide substitutions per site. B) Mean percentage of total identified interactions observed at 
184 each sample time point by phage RNR clade. Clade I phage RNR sequences were primarily 185 observed in late spring, while RNR sequences from Clades II and III were observed throughout 186 the summer. C) Aggregate network of Chesapeake Bay 'Cyano SP'-like phage-host interactions 187 from May - December 2018. Host taxonomy is colored as in panel A. Most 'Cyano SP'-like 188 phage RNR sequences were associated with a single Actinobacteria host 16S rRNA gene 189 identified as Luna-1 member Rhodoluna. with Actinobacteria (Fig. 4). Approximately 80\% (31/40) of the RNR sequences were linked to a single host classified as Actinobacteria Luna-1 subcluster member Rhodoluna, indicating this is

194 the primary host for these particular Chesapeake cyanopodoviral-like populations. Unlike 195 populations of Cyanobacteria (e.g. Synechococcus abundance varied $>1000$-fold between July 196 and December), this host displayed much less temporal variation in abundance (15-fold change

197 between July and December), which may explain the observed stability of putative

198 cyanopodoviral-like populations (1.1-fold change) compared to cyanomyoviruses (52-fold

199 change) in virome libraries across the same time-points. Screening single-amplified genome

200 (SAG) libraries further supports this observation. No Cyano SP-like RNR gene sequences could

201 be amplified from $~ 300$ Cyanobacteria SAGs from May 2017. In contrast, one Cyano SP-like

202 RNR gene sequence was associated with an Alphaproteobacteria SAG, while a second was

203 associated with an Acidimicrobiia (phylum Actinobacteria) SAG library (Fig. 4A), providing

204 further support that poorly characterized Chesapeake Bay viral populations with Cyano SP-like

205 RNR genes interact with Actinobacteria.

Actinobacteria, including Rhodoluna, were also commonly predicted hosts of

207 Chesapeake Bay viral populations using an in silico approach that infers the hosts through

208 compositional similarity between host and viral genomes $\left(\mathrm{WiSH}^{41}\right)$. The host genome database

209 was created from references genomes ${ }^{42}$ and metagenome assembled genomes (MAGs) from the 
210 environmental bacterial fractions. To identify significant host predictions, the viral database

211 included both viral populations and viral reference genomes. This resulted in significant $(\mathrm{p}<$

$2120.05)$ host predictions for about half (49\%) of the viral populations (Fig. S6). Most of the top

213 host predictions for Chesapeake Bay viral contigs were reference genomes (80\%); however, four

214 of the ten most frequently predicted hosts were Chesapeake Bay MAGs, all of which were

215 classified as Actinobacteria. In fact, among Chesapeake Bay MAGs Actinobacteria were the

216 predicted hosts for more viral contigs (57\%) than all other MAGs combined even though

217 Actinobacteria only comprised 25\% of MAG classifications overall (Fig. S6). Viral populations

218 with putative Chesapeake Bay Actinobacteria hosts were abundant and consistently observed

219 throughout the time series (Fig. S7), suggesting significant viral pressure on Actinobacteria

220 populations throughout the year.

221 Incubation experiments of water samples with and without active viruses from the same

222 site one year after the initial sample collection (July 2019) also suggest substantial viral pressure

223 on Actinobacteria lineages (Fig. S8). Actinobacteria lineages Acidimicrobiia and Actinobacteria

224 (class level) were the most commonly identified taxa susceptible to viruses, as determined by

225 differences in relative abundance between incubations with active and inactive virus fractions

226 (Fig. S8B). An identical 16S rRNA gene sequence to the primary Rhodoluna host identified by

227 epicPCR was in the top half of the most susceptible populations, while a sequence with one

228 nucleotide difference from the Rhodoluna host was in the top third. Previously, Actinobacteria

229 was the most frequently identified host for a set of over 2,000 freshwater phage genomes in an

230 analysis of freshwater viruses ${ }^{43}$, suggesting that Actinobacteria may be under substantial viral

231 pressure in both freshwater and estuarine environments. 


\section{In situ viral-host interaction dynamics reveal ecologically differentiated viral clades}

EpicPCR analysis revealed interaction patterns that suggest RNR viral clades are

235 ecologically differentiated. Phage-host interactions displayed seasonal patterns consistent with

236 those observed for the viral community as a whole. The total number of interactions identified

237 among Clade I 'Cyano SP'-like RNR phage sequences peaked in late May before largely

238 disappearing during the summer (Fig. 4B). Clade I phage sequences were most frequently

239 associated with multiple hosts (4/6 sequences) and the greatest number of hosts (Fig. 4A, C).

240 Host ranges for Clade I phages were significantly correlated with 'Cyano SP-like' phage

241 abundances measured by qPCR (Spearman's Rho $=0.69 ; \mathrm{p}<0.01$ ). Thus, the broader host

242 ranges of Clade I phages could be the temporary byproduct of higher viral abundances promoting

243 less efficient interactions through increased contact rates. Cultivation studies have shown that

244 bacterial hosts are not infected by different phages with equal efficiencies, as host sensitivity to

245 phage infection can differ by orders of magnitude at the same phage titre ${ }^{44}$. It is also possible that

246 broader host ranges provide an advantage for late spring viral populations under dynamic

247 conditions characterized by large freshwater influxes. 

summer (Fig. 4B). Most (7/8) Clade II phage sequences were associated with a single host, while

a.

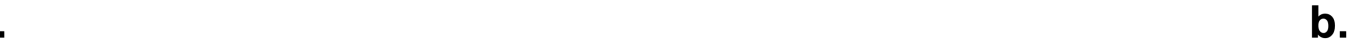

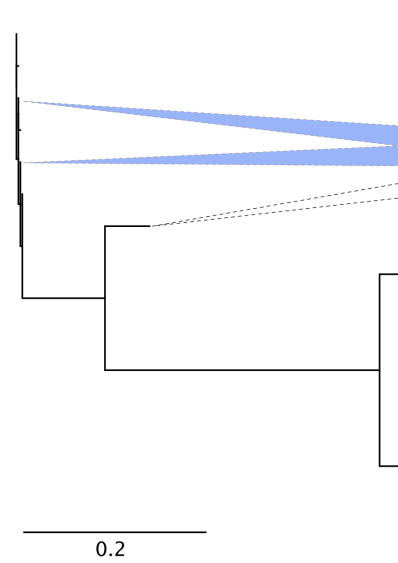

C.

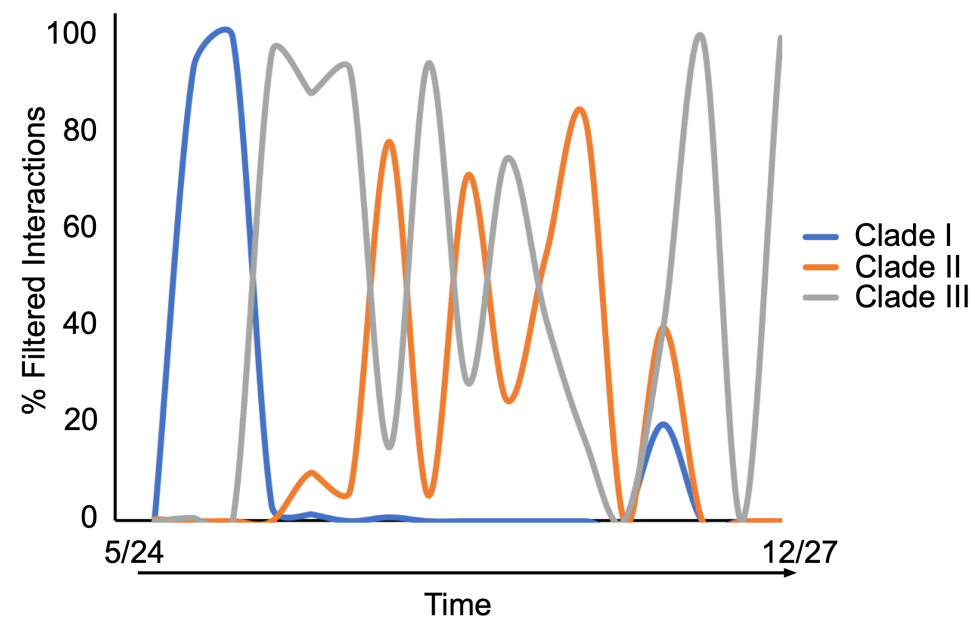

b.

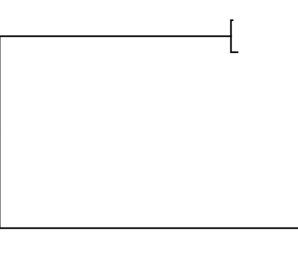

Time

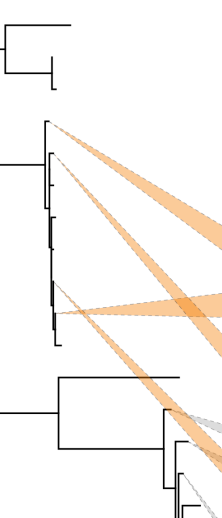

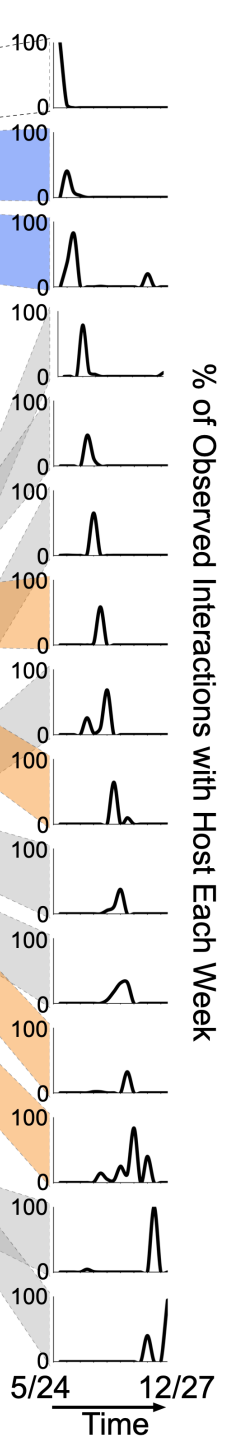

100

Figure 5. Phage interactions with Rhodoluna host observed from 5/24/18 to $12 / 27 / 18$. In total, 31 of 40 phage populations interacted with this host. A) Maximum likelihood tree with 100

253 bootstrap replicates of Chesapeake Bay phage ribonucleotide reductase (RNR) genes (G1380 to

254 A2079 in E. coli $\mathrm{nrdA}$ ). RNR gene sequences were clustered at $100 \%$ nucleotide identity prior to 255 phylogenetic analysis. Phage populations formed three main clades. Scale bar represents individual phage populations comprised each week. Colored wedges link phage populations to their corresponding dynamics over time. Wedge colors correspond to phage clades. Only the most abundant phage interactions were depicted for simplicity. C) Proportion of observed phage interactions with host over time by clade. Blue: Clade I; orange: Clade II; grey: Clade III. 
261 clade III sequences were more evenly split between single hosts (11/19 sequences) and multiple

262 hosts (8/19 sequences). Neither clade displayed the broad host ranges nor the correlation between

263 host range and viral abundance observed with Clade I phage sequences. During this time,

264 interactions between Rhodoluna and Clade II and Clade III phage populations alternated at most

265 time points (Fig. 5), which may be a reflection of tidal influences on phage and/or host diversity.

266 Supporting this hypothesis, Clade III interactions were negatively correlated with fluorescent

267 dissolved organic matter $($ FDOM) (Spearman's Rho $=0.67 ; \mathrm{p}<0.01)$, which can be strongly

268 influenced by tide at our sample site ${ }^{45}$, and positively correlated with salinity (Spearman's Rho

$269=0.57 ; \mathrm{p}<0.05)($ Table S2). This suggests Clade II and III phage populations may represent

270 riverine and estuarine diversity, respectively. However, we cannot rule out other possible

271 explanations, such as changes to host physiology with different environmental conditions ${ }^{46}$ or

272 antagonistic evolution between phage and host, as previously reviewed ${ }^{47}$.

273

Host range associated with different virus-host interaction lifespan and efficiency

Interactions captured by epicPCR illuminated differences between viral RNR sequences

276 associated with a single host (specialist phage) and multiple hosts (generalist phage) with regards

277 to in situ interaction persistence and interaction efficiency. Generalist phage RNR sequences

278 were observed in epicPCR fusion amplicons across a significantly $(\mathrm{p}<0.01)$ greater number of

279 sample dates than were specialist phage RNR sequences (Fig. 6A). This suggests that broader

280 host ranges may increase the 'interaction lifespan' of phage and could be an evolutionary

281 advantage in highly dynamic systems where viral infections appear to be largely ephemeral ${ }^{48,49}$

282 (Fig. 4). However, epicPCR and 16S rRNA marker gene analyses also revealed differences in

283 minimum host abundances when associated with generalist or specialist phages. Overall, 21 host 
sequences (100\% nucleotide identity) that were associated with generalist phages from epicPCR

287 (0.63\% mean). Eight hosts associated with specialist phages from epicPCR were also identified

288 in the $16 \mathrm{~S}$ rRNA libraries and had minimum abundances ranging from $0-0.33 \%$ of the

289 community ( $0.11 \%$ mean). Thus, the average minimum abundance of hosts when associated with

290 specialist phage RNR sequences was significantly $(\mathrm{p}<0.01)$ lower than when associated with

291 generalist phage RNR sequences (Fig. 6B). This could be attributed to increased infection

292 efficiencies among specialist phages compared to generalist phages. Consistent with these

293 findings, previous work identified fitness costs that were associated with increased host range in

294 cultivated phage-host systems ${ }^{50}$, and models of phage-host interactions suggest a trade-off in

295 viral infection efficiency with increasing host range ${ }^{12}$. Considering the differences we observed

296 in interaction persistence and interaction efficiency, there appears to be a trade-off between

297 'interaction lifespan' and infection efficiency for 'Cyano SP'-like phages in the Chesapeake Bay.

a.

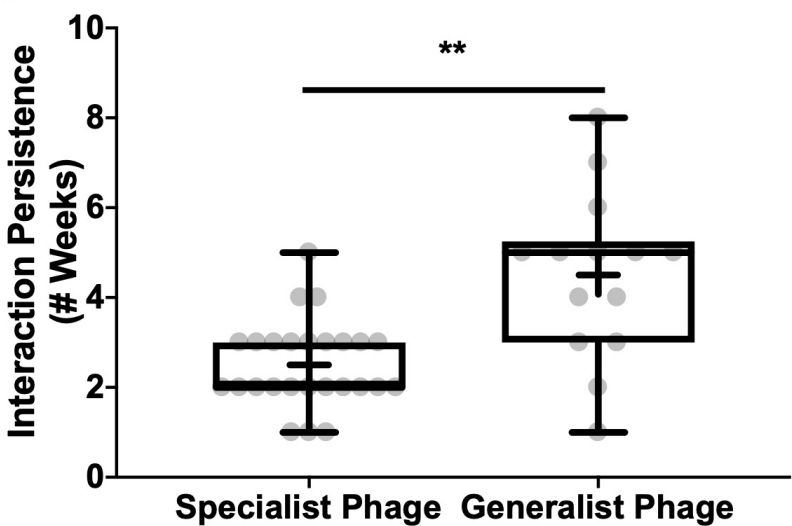

b.

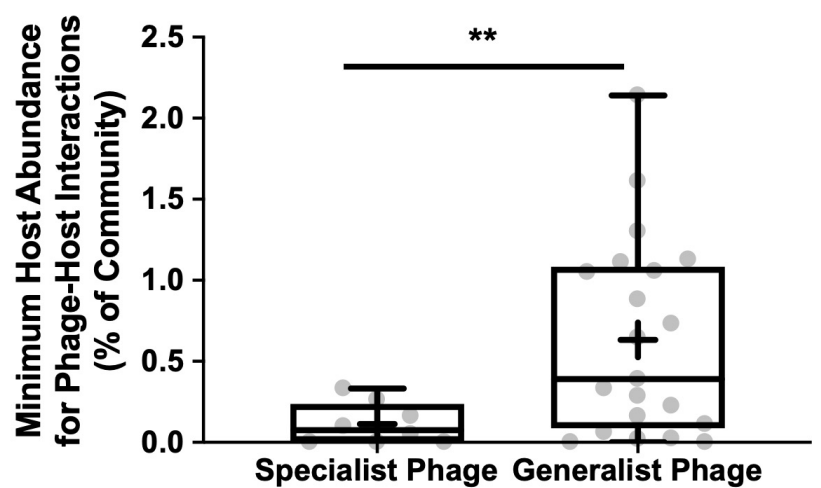

300 Figure 6. Biological trade-offs and ecological patterns related to viral and bacterial interactions.

301 A) Total interaction persistence for 'Cyano SP'-like phage RNR sequences associated with a

302 single host (specialist phage, $\mathrm{n}=26$ ) or multiple hosts (generalist phage, $\mathrm{n}=14$ ) between May

303 and December 2018 in the Chesapeake Bay $(* *=\mathrm{T}$ Test $\mathrm{p}<0.01)$. B) Minimum host 
abundance (16S rRNA gene relative abundance) of interactions with specialist phage RNR gene sequences $(n=8)$ and generalist phage RNR gene sequences $(n=21)$ observed in epicPCR fusion amplicons from samples collected between May and December 2018 in the Chesapeake Bay $(* *=\mathrm{T}$ Test $\mathrm{p}<0.01)$.

\section{Discussion}

EpicPCR fills a gap as a culture independent, high-throughput tool that can complement

311 existing methods in viral ecology by linking viral and bacterial populations in situ. EpicPCR can

312 complement large-scale metagenomic sequencing efforts ${ }^{51,52}$ through host identification for

313 novel and uncultivable viruses, a notable advantage over cultivation-based approaches, like high-

314 throughput methods like viral tagging ${ }^{53}$. The method also has a throughput advantage over

315 similar methods like digital PCR and single-cell genomics, which have also been applied to

316 identify host interactions ${ }^{22,54}$ but can currently only accommodate hundreds to a few thousand

317 wells for reactions and require costly and/or specialized equipment. In contrast, hundreds of

318 thousands of reactions are possible within an emulsion with epicPCR, and the method can be

319 performed using standard molecular biology equipment. Although primer bias remains an issue

320 for epicPCR, the viral marker gene could be modified to any of the commonly applied viral

321 marker genes ${ }^{55}$, or new primers could be developed to target novel viral diversity. For example,

322 epicPCR could be used to examine host ranges of abundant viruses in the ocean, such as vSAG

$32337-\mathrm{F} 6^{56,57}$, or to verify specific host predictions, such as for potential archaeal viruses ${ }^{58}$.

324 Recently, metagenomic time series have been used to predict environmental viral-host infection

325 networks ${ }^{59}$. Observations from epicPCR could inform and test the accuracy and underlying

326 assumptions of predicted network, improving our understanding of the drivers shaping microbial

327 diversity and function. Unlike cultivation-based methods or in silico predictions, epicPCR can

328 also capture virus-host interactions when and where they occur. This can be particularly useful 
329 for investigating how ecological and environmental factors influence these interactions. It should

330 be noted, however, that while epicPCR captures close physical contact consistent with infections,

331 it does not confirm active infections or differentiate between lytic or lysogenic infections, similar

332 to the limitations of single-cell genomics ${ }^{60}$. However, pairing epicPCR with metatranscriptomics

333 to examine expression of the viral marker gene ${ }^{48}$ could confirm active infections. Finally, pairing

334 the host range capabilities of epicPCR with quantitative methods of viral abundance, such as

$335 \mathrm{qPCR}^{61}, \mathrm{ddPCR}^{57}$ or polonies ${ }^{62}$, could be used to estimate the impact of viruses on different

336 microbial taxa and facilitate estimates of the viral influence in ecosystem-level models.

337 In this study, we developed primers to target a relatively well-studied group of

338 cyanophages and ascertain their in situ host interactions. This approach yielded unexpected host

339 associations and interaction dynamics. Our analysis revealed that these 'Cyano SP-like' RNR

340 populations commonly interact with Actinobacteria, which could not have been anticipated from

341 homology searches of any phage genomes known to be associated with cultured Actinobacteria

342 phage, such as within PhagesDB ${ }^{63}$. These associations are robust across methodologies, as

343 evidenced by the identification of an 'Cyano SP-like' RNR sequence within the DNA amplified

344 from a sorted, single cell with a 16S rRNA gene sequence that was classified as Actinobacteria.

345 In addition, some of the phages that interacted with Actinobacteria were also associated with

346 other hosts that spanned phyla. Although most viruses are believed to have narrow host ranges,

347 host enrichment may select against broad-host range phages ${ }^{64}$ and cultivated viruses capable of

348 infecting hosts spanning taxa at the order level have been reported ${ }^{65}$. Additionally, Paez-Espino

349 et al. (2016) ${ }^{66}$ found one percent of viruses from metagenomic libraries had predicted hosts that

350 spanned phyla based on CRISPR spacer and transfer RNA homology, including phages that were

351 associated with Actinobacteria. EpicPCR may be better suited to capture fleeting or inefficient 
352 associations with alternative hosts than other methods like plaque assays, where infection and

353 lysis by low-virulence phages can go undetected ${ }^{67}$, and could explain the greater frequency of

354 inter-phyla host ranges in this data set. Furthermore, cultivation-based host range experiments

355 often screen against related hosts. We most frequently observed generalist phages associated

356 with Rhodoluna (Actinobacteria) and the Nor5-3 clade of Gammaproteobacteria, which would be

357 unlikely to be screened together. It is also possible that these broad host ranges represent artifacts

358 of the epicPCR method even though we used controls in all environmental samples to remove

359 spurious interactions. However, observations of multiple different phages associated with the

360 same hosts (Rhodoluna and Nor5-3 Gammaproteobacteria) suggests this is unlikely.

$361 \quad$ EpicPCR enabled us to identify differences in interaction persistence and interaction

362 efficiency between generalist and specialist phage that would not have been identifiable in

363 shotgun sequence data, which can be used to test specific hypotheses, such as the impact of

364 generalist or specialist phages on community structure or the relative contribution of specific

365 interactions to viral production and bacterial mortality. Since generalist predators tend to have a

366 stabilizing effect on the overall community diversity at both the macro ${ }^{68}$ and micro scale ${ }^{69}$, we

367 would hypothesize that when Clade I infections are dominant, such as in the spring, they would

368 tend to stabilize community alpha diversity, while when Clade II infections are dominant, such

369 as with increasing amounts of FDOM, they would tend to destabilize diversity. These

370 interactions would be one in a myriad of interactions that would shape the overall community

371 diversity, but if these specific interactions had a disproportionate impact on the community-level

372 processes, the stabilizing and destabilizing effects might be observable at the community level.

373 Virus-host interaction dynamics revealed by epicPCR shed light on the individual interactions

374 that contribute to viral production and bacterial mortality over time and with changing 
375 conditions. Previous studies revealed differences in viral productivity and bacterial mortality

376 associated with spring and neap tides ${ }^{8}$. While our study did not quantify viral productivity or

377 bacterial mortality, we did find that interactions between Rhodoluna and Clade II and Clade III

378 phages were associated with salinity changes. Advances to this technique, such as pairing with a

379 microfluidics-based droplet maker and sorting and uniquely barcoding positively amplified

380 droplets may yield quantitative results, although these advances would limit wide-spread

381 accessibility. In this way, such quantitative measures could be paired with measurements of viral

382 productivity and bacterial mortality to determine the relative contribution of specific interactions

383 to viral production and bacterial mortality.

\section{Conclusions}

Viruses influence coastal ocean and estuarine biogeochemistry through interactions with

387 hosts mediating key autotrophic and heterotrophic processes. Using a combination of shotgun

388 metagenomics, marker gene analysis, dilution experiments and a novel gene fusion technique,

389 we investigated the factors that influence virus-host interactions within the largest estuary in the

390 United States. This estuary has a unique and endemic viral community that varies more between

391 seasons than between years. We found Actinobacteria populations are under substantial viral

392 pressure within this ecosystem and their interactions with specific phage clades seem to vary

393 with tidally- influenced environmental factors. The observations of virus-host interactions from

394 epicPCR also suggest that single- and multi-host phages have substantial differences in their

395 'interaction lifespan' and realized host abundance characteristics. This combination of

396 bioinformatics and experimental approaches provided high genetic and temporal resolution of

397 viral interactions with one of the most abundant heterotrophic bacterial populations within this 
ecosystem that can be widely applied across aquatic ecosystems to gain insight into viral

399 ecology.

400

401 Materials and Methods

402 Sample Collection and preservation

403 Surface water samples were collected from the mouth of the Rhode River (Edgewater,

404 MD) off of the Smithsonian Environmental Research Center (SERC) research pier. Samples

405 were collected five times a day over three days from $05 / 17 / 17-05 / 19 / 17$, between $10: 30$ am and 4:30 pm. Samples were also collected at 12:00 pm weekly from 5/24/18 to $8 / 9 / 18$, on $8 / 23 / 18$,

407 then again weekly from $12 / 6 / 18$ to $12 / 27 / 18$. Briefly, $25 \mathrm{~mL}$ of water sample was combined with $40825 \mathrm{~mL}$ of $50 \%(\mathrm{v} / \mathrm{v})$ sterile glycerol. Glycerol samples were stored on dry ice for transport back 409 to Baltimore, MD and subsequently stored at $-80^{\circ} \mathrm{C}$ until processing. Approximately $120 \mathrm{~mL}$ of

410 water sample was also collected per time point and filtered through a $0.2 \mu \mathrm{m}$ PES membrane

411 filter (Millipore, Inc.) for shotgun metagenomic analyses. Filters were stored on ice for transport

412 back to Baltimore, $\mathrm{MD}$ and kept at $-80^{\circ} \mathrm{C}$ until processing. Viral filtrates $(<0.2 \mu \mathrm{m}$ fraction $)$

413 were collected and incubated with $\mathrm{FeCl}_{3}$ as previously described ${ }^{70}$ during transport back to

414 Baltimore, MD. Viral filtrates were filtered through a $0.2 \mu \mathrm{m}$ PES membrane filter (Millipore,

415 Inc.) post-incubation, and filters were stored in the dark at $4^{\circ} \mathrm{C}$ until processing for shotgun

416 sequencing. Water conditions were recorded from the continuous water monitoring station

417 located at SERC (http://nmnhmp.riocean.com, Table S3). 
421 curve of gene copy number, viral 'Cyano SP'-like RNR genes were amplified from

422 environmental samples using primers Cyano_II_F and Cyano_II_R (0.3 uM final concentration

423 each, Table S4). Amplicons were run on a 1.5\% agarose gel and visualized with SYBR Safe

424 DNA gel stain (Invitrogen, 1x final concentration). RNR bands were cut out, gel purified (Zymo,

425 Inc.), and cloned into chemically competent Escherichia coli cells using the Zero Blunt PCR

426 Cloning Kit (Thermo Scientific) following the manufacturer's protocol. Cells were grown

427 overnight on $\mathrm{LB}+$ kanamycin $\left(50 \mu \mathrm{g} \mathrm{mL} \mathrm{m}^{-1}\right)$ plates at $37^{\circ} \mathrm{C}$ and colonies were picked for

428 subsequent testing. Picked colonies were tested for amplification of the RNR gene. One colony

429 was serially diluted and grown on plates to determine gene copy number per dilution. The serial

430 dilution series was used to create a standard curve for use in qPCR. All standards and

431 environmental samples were run in triplicate. Three microliters of sample were combined with

432 UltraPure molecular grade water (Thermo, Inc.), SsoAdvanced Universal SYBR Green

433 Supermix (1x final concentration, Bio-Rad Laboratories, Inc.), Cyano_II_F primer (0.3 $\mu \mathrm{M}$ final

434 concentration), and Cyano_II_R primer (0.3 $\mu \mathrm{M}$ final concentration) to a final volume of $25 \mu \mathrm{L}$

435 (see Table S4 for primer sequences and citations). Samples were amplified on a CFX96 Real-

436 Time PCR Detection System (Bio-Rad Laboratories, Inc.) with the following conditions:

437 denaturing at $98^{\circ} \mathrm{C}$ for 10 minutes; 45 cycles of denaturing at $98^{\circ} \mathrm{C}$ for 10 seconds, annealing at

$43852^{\circ} \mathrm{C}$ for 30 seconds, and extension at $72^{\circ} \mathrm{C}$ for 45 seconds; and a final extension of $72^{\circ} \mathrm{C}$ for 5

439 minutes. The same dilution series was used to create a standard curve for the 16S rRNA gene.

440 16S rRNA gene counts were assessed as above with primers PE_16S_U515F and 16S_1114R

441 (Table S4). Bacterial cell counts in environmental samples were predicted based on the gene 
442 counts and copy number correction between E. coli (7 copies) and aquatic bacteria

443 (approximately 3 copies).

Shotgun metagenomic library preparation, sequencing, and processing

447 the following dates: 05/17/17, 05/18/17, 05/19/17, 05/31/2018,06/28/18,08/02/18, and 12/6/18.

448 Additionally, two positive controls were processed, a Zymo positive control community (Zymo

449 Research) and E. coli, and one negative control (water). DNA extraction was performed with the 450 DNeasy PowerWater kit (Qiagen) following the manufacturer's protocol with the following

451 amendment: $20 \mu \mathrm{L}$ of proteinase $\mathrm{K}$ was combined with $1 \mathrm{~mL}$ of solution PW1 in the bead tube.

452 The bead tube was incubated at $65^{\circ} \mathrm{C}$ for ten minutes prior to bead beating. Libraries were

453 prepared with the Nextera DNA Flex Library Prep kit (Illumina, Inc.) following the

454 manufacture's protocol and sequenced on an Illumina MiSeq ( 2 x $300 \mathrm{bp})$ at the Genetic Core 455 Research Facility at Johns Hopkins University.

456 Sequences were quality filtered and trimmed with trimmomatic (v. 0.38$)^{71}$, assembled 457 with metaSPAdes (v. 3.13.1) ${ }^{72}$. The assembly was used to generate metagenome assembled 458 genomes (MAGs) through metaWRAP ${ }^{73}$ with metabat ${ }^{74}$ and maxbin $2^{75}$. Binning_refiner ${ }^{76}$ was

459 used to create the final set of MAGs with at least 50\% completeness and less than $10 \%$

460 contamination, as determined by CheckM $^{77}$. MAGs were classified using Kaiju ${ }^{78}$ 
465 column-purified DNA were combined with UltraPure molecular grade water (Thermo, Inc.), 10X

466 buffer (1x final concentration), dNTPs (0.1mM each final concentration), 16S forward primer

467 27F (0.3 $\mu \mathrm{M}$ final concentration), 16S reverse primer PE_16S_V4_E786_R (0.3 $\mu \mathrm{M}$ final

468 concentration), bovine serum albumin ( $0.02 \mathrm{mg} / \mathrm{mL}$ final concentration), and Phusion High-

469 Fidelity DNA Polymerase (0.5U; New England BioLabs, Inc.; see Table S4 for primer sequences

470 and citations). PCR reactions were combined with $150 \mu \mathrm{L}$ of $4 \%$ UMIL EM90 oil (4\% UMIL

471 EM90 oil, 0.05\% TritonX-100 v/v in mineral oil; Universal Preserv-A-Chem, Inc.) and

472 emulsified by vortexing at max speed $(\sim 2,700 \mathrm{rpm})$ for one minute on a Vortex Genie 2

473 (MoBio). Emulsions were loaded as $50 \mu \mathrm{L}$ aliquots and amplified with the following conditions:

474 denaturation at $94^{\circ} \mathrm{C}$ for 3 minutes; 33 cycles of denaturation at $94^{\circ} \mathrm{C}$ for 10 seconds, annealing

475 at $54^{\circ} \mathrm{C}$ for 30 seconds, and extension at $72^{\circ} \mathrm{C}$ for 45 seconds; and a final extension of $72^{\circ} \mathrm{C}$ for 5

476 minutes (C1000, BioRad Labs., Inc.). Samples were immediately removed upon completion of

477 amplification and stored at $-20^{\circ} \mathrm{C}$ until the emulsion was broken.

PCR oil emulsions were broken with isobutanol as previously described ${ }^{79}$. Briefly, PCR

479 aliquots were pooled in a $1.5 \mathrm{~mL}$ microcentrifuge tube and combined with $100 \mathrm{uL}$ of sterile $5 \mathrm{M}$

$480 \mathrm{NaCl}$ solution and $1 \mathrm{~mL}$ of isobutanol. Samples were vortexed briefly to mix and centrifuged at

$48116,000 \times \mathrm{g}$ for 1 minute. The bottom aqueous layer was retained, and DNA was purified by spin

482 column purification (Zymo, Inc.). DNA was eluted in $20 \mathrm{uL}$ of Tris- $\mathrm{HCl}$ and stored at $-20^{\circ} \mathrm{C}$.

483 Purified DNA was run on a 1.5\% agarose gel (UltraPure Agarose, ThermoFisher

484 Scientific) and visualized with SYBR Safe DNA gel stain (Invitrogen, 1x final concentration).

485 The gel was run in $1 \mathrm{X}$ TBE buffer (Alfa Aesar) at $4 \mathrm{~V} / \mathrm{cm}$. 16S rRNA gene bands were

486 visualized under blue light excitation, extracted, and gel purified (Zymo, Inc.) Purified DNA was

487 eluted into $20 \mu \mathrm{L}$ of Tris- $\mathrm{HCl}$ and stored at $-20^{\circ} \mathrm{C}$ until further processing. 

subsequent limited PCR steps. Barcodes were added as follows: two microliters of purified DNA were combined with UltraPure molecular grade water (Thermo, Inc.), 10X buffer (1x final

491 concentration), dNTPs (0.1mM each final concentration), 16S forward primer

492 PE_16S_V4_U515F (0.3 $\mu \mathrm{M}$ final concentration), 16S rRNA gene reverse primer with 8-mer

493 barcodes PE_IV_XXX (0.3 $\mu \mathrm{M}$ final concentration), and Phusion High-Fidelity DNA

494 Polymerase (0.5U; New England BioLabs, Inc.; see Table S4 for primer sequences and 495 citations). Samples were amplified with the following conditions: denaturing at $98^{\circ} \mathrm{C}$ for 30

496 seconds; 8 cycles of denaturing at $98^{\circ} \mathrm{C}$ for 10 seconds, annealing at $54^{\circ} \mathrm{C}$ for 30 seconds, and 497 extension at $72^{\circ} \mathrm{C}$ for 45 seconds; and a final extension of $72^{\circ} \mathrm{C}$ for 5 minutes. DNA was purified 498 by spin column purification (Zymo, Inc.) and eluted into $20 \mu \mathrm{L}$ Tris-HCl. Illumina adapters were 499 then added as above with the following primers: Illumina adapter forward primer PE-III-PCR-F

$500(0.3 \mu \mathrm{M}$ final concentration) and Illumina adapter reverse primer Barcode_Rev $(0.3 \mu \mathrm{M}$ final 501 concentration) (see Table S4 for primer sequences and citations). Samples were amplified with 502 the following conditions: denaturing at $98^{\circ} \mathrm{C}$ for 30 seconds; 5 cycles of denaturing at $98^{\circ} \mathrm{C}$ for 50310 seconds, annealing at $54^{\circ} \mathrm{C}$ for 30 seconds, and extension at $72^{\circ} \mathrm{C}$ for 45 seconds; and a final 504 extension of $72^{\circ} \mathrm{C}$ for 5 minutes. DNA was purified by spin column purification (Zymo, Inc.) 505 and eluted into $20 \mu \mathrm{L}$ Tris-HCl. 16S rRNA gene amplicon products were quantitated on a Qubit 3.0 fluorometer 507 (Invitrogen) and three nanograms of DNA pooled per sample for sequencing. 16S rRNA gene 508 amplicon libraries were sequenced on an Illumina MiSeq (2 x $300 \mathrm{bp})$ at the Genetic Core 509 Research Facility at Johns Hopkins University. Sequence reads were processed in QIIME2 ${ }^{80}$ 
510 using the DADA2 de-noising pipeline. Taxonomic assignment was performed in QIIME2 with

511 the Greengenes ${ }^{81}$ database.

513 Virome shotgun metagenomic library preparation, sequencing, and processing

514 Samples from the following dates were used for virome short-read shotgun metagenomic

515 analysis: $05 / 17 / 17,05 / 18 / 17,05 / 31 / 18,08 / 02 / 18,12 / 14 / 18,12 / 20 / 18$. The two samples collected

516 on 12/14/18, 12/20/18 were also long-read sequenced. Viruses were resuspended from filters

517 with an ascorbic acid buffer as previously described ${ }^{70}$. Following resuspension, viral particles

518 were purified by cesium chloride gradient centrifugation ${ }^{82}$ and DNA extracted with Wizard Prep

519 Columns (Promega, Corp.). Viral metagenome short-read libraries were prepared using the

520 NexteraXT kit (Illumina, Inc.) following the manufacturer's protocol. For samples with $>0.16$

$521 \mathrm{ng} / \mu \mathrm{L}$, samples were amplified with 15 cycles; for those with 0.1-016 ng/ $\mathrm{L}$, samples were

522 diluted 1:5 and amplified with 18 cycles; for $<0.1 \mathrm{ng} / \mu \mathrm{L}$, samples were diluted 1:10 and

523 amplified with 20 cycles. Short reads for all virome libraries were sequenced on an Illumina

524 Short reads for all virome libraries were sequenced on an Illumina NovaSeq S4 with 75M

525 (target) 2x150bp reads at the JP Sulzberger Genome Center (Columbia University, New York,

526 NY). Additionally, the long-read libraries from December 2018 were prepared using

527 phenol:chloroform extraction protocol (dx.doi.org/10.17504/protocols.io.6cbhasn) and libraries

528 were prepared as previously described ${ }^{24}$ with modifications and sequenced with an Oxford

529 Nanopore MinION instrument on a FLO-MIN106D R9 version Spot-ON flowcell (Rev D) at the

530 Ohio State University according to the manufacturer's instructions.

531 Short reads were cleaned and quality-trimmed with bbduk ${ }^{83}$; adapters, sequencing

532 artifacts, and PhiX sequences were removed (ktrim=r; k=23 mink=11; hdist=1; hdist2=1). Reads 
533 were then quality-trimmed from both ends to remove bases with low quality scores (qtrim=rl;

534 trimq=20). Reads shorter than 30 bp (minlength=30), with Ns (maxns=0), or with an average

535 quality below $20(\mathrm{maq}=20)$ were discarded. The cleaned reads from each sample were then

536 independently assembled with metaSPAdes (v. 3.13.1) ${ }^{72}$ using k-mer sizes: 21, 33, 55, 77, and -

537 meta parameter. Long-reads were basecalled with Guppy v.2.3.1 (Manufacturer's tool) and

538 individual barcoded sample libraries were demultiplexed with the 'barcoder' function of Guppy.

539 Long-reads were quality controlled with NanoFilt ${ }^{84}$, in which reads were filtered by quality

540 score (Q-score $\geq 10)$ and minimum length $(\geq 1 \mathrm{~kb})$, and finally 'headcropped' by $50 \mathrm{bp}$ to ensure

541 no remaining barcode sequence remained. These cleaned long-reads were used in two separate

542 assembly scenarios. First, long-reads were assembled with Flye ${ }^{85}$ in metagenomic mode (--

543 meta). Error-correction of the Flye assemblies where performed with Pilon v.1.23 ${ }^{86}$, using the

544 corresponding short-reads mapping information [read recruitment was performed with BWA

545 v.0.7.17 ${ }^{87}$ ] to detect and reduce basecalling errors. Second, hybrid assembly, using both long-

546 and short-reads was performed with SPAdes (v.3.13.1) ${ }^{72}$. Long reads were assembled through

547 hybrid assembly (with metaSpades hybrid option) and Flye ${ }^{85}$. In order to predict viral contigs in

548 the assembled datasets, assemblies were run through VirSorter ${ }^{88}$ (v. 1.0.5) in the -virome mode

549 after upgrading its database with an expanded profile hmm database of viral proteins, mainly

550 from the GOV2.0 dataset ${ }^{14}$. Contigs that were resolved as categories 1, 2, 4 and 5 were retained,

551 and filtered by length $\geq 5 \mathrm{~kb}\left(\geq 1.5 \mathrm{~kb}\right.$, if circular). DeepVirFinder ${ }^{89}$ was another tool for rescuing

552 additional viral contigs. We considered high-confidence viral contigs from DeepVirFinder to be

553 those of scores of $\geq 0.9$ with a $\mathrm{p}$-value $\leq 0.05$, and lengths $\geq 5 \mathrm{~kb}$. These two sets of predicted

554 viral contigs (from both long- and short-read assemblies) were then dereplicated using 
555 "ClusterGenomes" ${ }^{90}$ into viral populations using $95 \%$ average nucleotide identity over $80 \%$

556 coverage of the shorter contig length.

557 To calculate the coverages of these viral populations, clean reads were mapped to the

558 Chesapeake Bay viral population database with Bowtie ${ }^{91}$ in the non-deterministic and

559 sensitive mode. The output bam files were parsed using BamM

560 (https://github.com/Ecogenomics/BamM) to only keep the reads that covered over $70 \%$ of the

561 viral contig length, with over 75\% read alignment length. Pysam (https://github.com/pysam-

562 developers/pysam) was then used to filter out reads with $<95 \%$ identity. Trimmed pileup

563 coverage "tpmean" for each contig was calculated using BamM and then they were adjusted for

564 each sample by metagenome size. The same read mapping strategy was employed for RNR

565 sequences upon constructing the rank abundance curves in (Fig. S10)

566 To study seasonal diversity of Chesapeake Bay viral populations, all viromes were

567 randomly subsampled to $15 \mathrm{M}$ reads using bbmap "reformat" ${ }^{83}$ with default parameters. The

568 subsampled read libraries were assembled using metaSPAdes (v. 3.13.1) as above. The resulting

569 assemblies were then processed with VirSorter and DeepVirFinder as above. Viral contigs

570 extracted using the same cutoffs as mentioned before were grouped into viral populations if they

571 shared $\geq 95 \%$ nucleotide identity across $\geq 80 \%$ of the shorter contig length. Subsequently, the

572 subsampled reads were recruited to the viral populations' representatives with Bowtie2, and the

573 same read-mapping cutoffs discussed above were applied to calculate sequence-depth adjusted

574 coverage. Taxonomic assignment of viral populations was performed using vConTACT2 90,92

575 with default parameters. First, the full proteome of every viral population representative was

576 predicted using prodigal ${ }^{93}$. The protein set was then combined with all the proteins from the

577 phage and archaeal viruses in the NCBI RefSeq v88 release. The combined set of proteins was 
578 then used as input for vConTACT2 to compute protein similarity overlaps (i.e. protein clusters),

579 and subsequently refined into genus-level equivalent 'viral clusters' (VCs). Using this method,

580 viruses are classified at the genus level. The resulting cluster file were imported and visualized in

581 Cytoscape 3.7.2.

582

583 Seasonal dynamics and diversity analyses

Seasonal bacterial communities were assessed from 16S rRNA gene amplicon libraries.

Hierarchical clustering was performed in QIIME ${ }^{95}$ with Bray-Curtis dissimilarity. Libraries were sub-sampled at 150,000 observations with ten jackknifed replicates. Community structure was

587 visualized using heatmap. 2 in the gplots ${ }^{96}$ package in $\mathrm{R}$.

Seasonal Chesapeake Bay viral communities were characterized by mapping reads to assembled contigs using Bowtie $2^{97}$ with default sensitive conditions. To compare viral populations with a deeply sequenced virome library collected in 2012 at the same location ${ }^{36}$,

5912017 and 2018 Chesapeake Bay virome reads were mapped to both the $>5 \mathrm{~kb}$ contigs assembled

592 in this study and contigs $>5 \mathrm{~kb}$ from the 2012 virome that were previously assembled. Only reads

593 that mapped to contigs with $\geq 90 \%$ identity over $\geq 90 \%$ of the read were retained. Viral

594 populations were considered present in the sample if retained reads mapped to $\geq 75 \%$ of the

595 contig. Trimmed pileup coverage values were used as proxies of observation counts for each

596 population. Hierarchical clustering was performed in QIIME with Bray-Curtis dissimilarity.

597 Libraries were sub-sampled at 300,000 observations with ten jackknifed replicates. Community

598 structure was visualized in $\mathrm{R}$ as described above.

599 Shannon diversity was calculated in QIIME for libraries with corresponding virome 600 samples $(5 / 17 / 2017,5 / 31 / 2018,8 / 2 / 2018,12 / 14 / 2018,12 / 20 / 2018)$. Values were recorded as the 
601 average of ten jackknifed replicates at a sampling depth of 10,000 observations for both 16S and 602 viral populations.

The distribution of Chesapeake Bay viral populations in ocean viral metagenomes was

604 investigated by mapping viral metagenome reads from Global Ocean Virome 2.0 database

605 libraries ${ }^{14}$ to the Chesapeake Bay $>5 \mathrm{~kb}$ contigs as described above for the Chesapeake Bay 2012

606 viral metagenome sample. Additional libraries from previously published freshwater and

607 estuarine environments were similarly queried ${ }^{29-31}$, in addition to a viral metagenome from the

608 Damariscotta River Estuary, ME, USA (BioProject Accession No. PRJNA357591).

Ribonucleotide reductase homology analysis

612 MetaGeneMark $^{98}$. Ribonucleotide reductase (RNR) alpha subunit genes were identified by

613 BLASTx query of ORFs (e-value 1E-10) against a database of Uniref50 ${ }^{99}$ RNR cluster

614 representative sequences. Putative RNR genes were translated to amino acid sequences and

615 aligned with MAFFT $^{100}$. Aligned sequences were visually inspected in Geneious ${ }^{101}$ v. 9.1.5 for

616 the presence of conserved catalytic residues C439, E441, C462 indicative of RNR proteins

617 (amino acid positions of Escherichia coli nrdA gene product). Only sequences containing these

618 conserved amino acids and spanning the C462 to P621were retained. All retained sequences

619 were queried against viral sequences in the NCBI nr database by BLASTn to identify putative

620 viral and host taxonomy. Top hits with a percent identity $\geq 65 \%$ across at least $90 \%$ of the query

621 sequence were recorded. All sequences with top hits below this threshold were reported as

622 'Unclassified'. 
Bioinformatics host prediction with WIsH

626 length. The entire dataset used in the WIsH analysis included viral contigs and metagenome

627 assembled genomes (MAGs $>50 \%$ completion, $<10 \%$ contamination) from the Rhode River,

628 along with the viral and hosts reference genomes from the WIsH benchmark dataset, downloaded

629 from NCBI. Because an appropriate null model is not available for this unique estuarine

630 environment, we assume that for every bacterial model, the set of phage genomes in the dataset

631 for which it is a host is negligible compared to the set of phage genomes that for which it is not a

632 host. Reference host and viral genome relationships in the benchmark dataset were used to verify

633 the performance under these assumptions. Running the benchmark dataset with the null model

634 yielded similar results as those previously reported (58\%, as compared to $63 \%$, accuracy

635 reported at the genus level). Thus, we applied the same assumption for the null model to the

636 analysis of environmental data. The analysis required top hits to have a likelihood value in the

637 top $5 \%$ of all calculated likelihoods $(\mathrm{p}<=0.05)$. Of the 9,392 viral contigs from the Chesapeake

638 Bay, almost half had top predictions that were within the top 5\% of all tested likelihoods. Of

639 significant hits, $80 \%$ of top predictions of hosts for environmental viral population mapped to

640 reference genomes and $20 \%$ of significant top predicted hosts were MAGs. 
647 the time points. Samples were centrifuged at 25,000 x g for 10 minutes and resuspended after

648 supernatant removal to reduce free viral particles. Thirty microliters of each sample was

649 combined with UltraPure molecular grade water (Thermo, Inc.), 10X buffer (1x final

650 concentration), dNTPs (0.1mM each final concentration), Cyano_SP_F primer (1.0 $\mu \mathrm{M}$ final

651 concentration), Cyano_SP_R_519R primers (0.01 $\mu \mathrm{M}$ each final concentration), S-*_Univ-1100-

652 a-A-15 $16 \mathrm{~S}$ reverse primer $(1.0 \mu \mathrm{M}$ final concentration $)$, bovine serum albumin $(0.02 \mathrm{mg} / \mathrm{mL}$

653 final concentration), Tween-20 (0.8\% v/v final concentration), and Phusion High-Fidelity DNA

654 Polymerase (1.5U; New England BioLabs, Inc.) to a final volume of $75 \mu \mathrm{L}$ (see Table S4 for

655 primer sequences and citations). PCR reactions were combined with $450 \mu \mathrm{L}$ of $4 \%$ UMIL EM90

656 oil (4\% UMIL EM90 oil, 0.05\% TritonX-100 v/v in mineral oil; Universal Preserv-A-Chem,

657 Inc.) and emulsified by vortexing at max speed $(\sim 2,700 \mathrm{rpm})$ for one minute. Emulsions were

658 loaded as $50 \mu \mathrm{L}$ aliquots and amplified with the following conditions: denaturation at $94^{\circ} \mathrm{C}$ for 3

659 minutes; 33 cycles of denaturation at $94^{\circ} \mathrm{C}$ for 10 seconds, annealing at $54^{\circ} \mathrm{C}$ for 30 seconds, and

660 extension at $72^{\circ} \mathrm{C}$ for 45 seconds; and a final extension of $72^{\circ} \mathrm{C}$ for 5 minutes $(\mathrm{C} 1000$, BioRad

661 Labs., Inc.). Samples were immediately removed upon completion of amplification and stored at

$662-20^{\circ} \mathrm{C}$ until the emulsion was broken as described above.

663

664 Preventing fusion of unfused genes during nested PCR

665

Blocking primers were used during nested PCR to prevent the annealing and

666 amplification of unfused genes from the emulsion PCR as previously described ${ }^{23}$. The efficacy of

667 the blocking primers was tested to ensure random virus-host sequences were not being

668 introduced. First, RNR and 16S rRNA gene copy numbers were quantitated in all epicPCR

669 reactions post-cleanup by qPCR as described above. Next, RNR genes were amplified from 
670 Rhode River samples with primers Cyano_II_F and Cyano_II_R_519R primers $(0.3 \mu \mathrm{M}$ final

671 concentration each, Table S4). 16S rRNA genes were similarly amplified with primers 27F and

672 1492R (0.3 $\mu \mathrm{M}$ final concentration each, Table S4). Unfused RNR and 16S rRNA gene

673 amplicons were combined at copy numbers equal to the highest observed across all samples after

674 epicPCR as determined by qPCR. Finally, the unfused RNR and 16S rRNA gene mixture was

675 run through nested PCR and barcoding PCR reactions with all environmental samples as

676 described below. Samples were run on a 1.5\% agarose gel and visualized with SYBR Safe DNA

677 gel stain (Invitrogen, 1x final concentration). Fusion products were observed in the unfused mix

678 control when no blocking primers were used. However, with the addition of blocking primers no

679 fusion products were detected in the unfused mix control.

681 Enrichment of viral-host fused amplicons

683 DNA were combined with UltraPure molecular grade water (Thermo, Inc.), 10X buffer (1x final

684 concentration $),$ dNTPs $(0.1 \mathrm{mM}$ each final concentration), Cyano_SP_Nested_FA primer $(0.3 \mu \mathrm{M}$

685 final concentration), 16S reverse primer PE_16S_V4_E786_R (0.3 $\mu \mathrm{M}$ final concentration),

686 forward and reverse blocking primers U519F-block10 and U519R-block10 (1.0 $\mu \mathrm{M}$ final

687 concentration each; to ensure no amplification of unfused genes, see above), and Phusion High-

688 Fidelity DNA Polymerase (0.5U; New England BioLabs, Inc.) to a final volume of $25 \mu \mathrm{L}$ (see

689 Table S4 for primer sequences and citations). Samples were amplified with the following

690 conditions: denaturing at $94^{\circ} \mathrm{C}$ for 30 seconds; 30 cycles of denaturing at $94^{\circ} \mathrm{C}$ for 10 seconds,

691 annealing at $54^{\circ} \mathrm{C}$ for 30 seconds, and extension at $72^{\circ} \mathrm{C}$ for 45 seconds; and a final extension of

$69272^{\circ} \mathrm{C}$ for 5 minutes. PCR reactions were cleaned by spin column purification (Zymo, Inc.) and 
693 DNA eluted in $20 \mathrm{uL}$ of Tris-HCl. Samples were barcoded following enrichment and cleanup by

694 PCR as described above with Cyano_SP_Nested_FB primer $(0.3 \mu \mathrm{M}$ final concentration, Table

695 S4), reverse primer PE-IV-PCR-XXX with 8-mer barcodes (0.3 $\mu \mathrm{M}$ final concentration, Table

696 S4), and forward and reverse blocking primers U519F-block10 and U519R-block10 (1.0 $\mu \mathrm{M}$

697 final concentration each, Table S4). Barcoded samples were run on a 1.5\% agarose gel

698 (Invitrogen) and visualized with SYBR Safe DNA gel stain (Invitrogen, 1x final concentration).

699 Fusion bands were cut out and gel purified (Zymo, Inc.). Fusion products were quantitated on a

700 Qubit 3.0 fluorometer (Invitrogen) and ten nanograms of DNA pooled per sample for

701 sequencing.

702

703 Sequencing and quality filtering of viral-host fusion amplicons

704

Fused amplicon products were sequenced on a PacBio Sequel with Sequel v3 chemistry

705 (University of Maryland Institute for Genome Sciences). Circular consensus sequences were

706 obtained from raw reads with the following parameters: minimum signal-to-noise ratio (SNR): 3,

707 minimum length: $500 \mathrm{bp}$, minimum passes: 10 , minimum read score: 0.75 , minimum predicted

708 accuracy: 0.90. Consensus sequences passing these thresholds were oriented by searching for

709 primer sequences using Cutadapt ${ }^{102}$ with an error rate of 0.01 . Only sequences passing this error

710 rate were retained. Following orientation, reads were demultiplexed in QIIME ${ }^{95}$ with zero

711 allowed barcode mismatches. Fused gene products were split by gene and primer sequences

712 identified with Cutadapt ${ }^{102}$. Only those sequences without mismatches in any primer sites (error

713 rate of 0.01 ) were retained for further analyses. Finally, amplicons were filtered by size to

714 remove truncated or chimeric reads. Only fusion sequences with RNR gene amplicons between

715583 and 596bp and 16S rRNA gene amplicons between 249 and 262bp were retained. These 
716 sequences were split into separate RNR and 16S genes and their primer sequences trimmed with

717 Cutadapt $^{102}$.

719 Analyzing viral and bacterial diversity from epicPCR fusion amplicons

$720 \quad$ Viral RNR and bacterial 16S rRNA gene sequences passing all quality filtering were

721 clustered at $100 \%$ nucleotide identity with CD-HIT ${ }^{103}$ to identify viral and bacterial populations.

722 Only viral-host pairs observed in three or more libraries were considered positive interactions.

723 Representative sequences from these viral and bacterial populations were queried against the

724 NCBI nr database via BLASTn to identify top hits. RNR gene sequences were aligned with

725 MAFFT $^{100}$ using the L-INS-I setting. Sequences were trimmed to the region G1380 to A2079 in

726 E. coli $n r d A$ and a maximum likelihood tree with 100 bootstrap replicates was made using

727 Phylogeny.fr (http://phylogeny.lirmm.fr) ${ }^{104}$. Viral-host interaction networks were visualized in

728 Cytoscape.

729

Viral dilution incubation experiments

731 One liter of surface water was collected in July 2019 from the mouth of the Rhode River

732 (Edgewater, MD) at the Smithsonian Environmental Research Center (SERC). 120mL was

733 filtered through two $0.2 \mu \mathrm{m}$ PES membrane filter (Millipore, Inc.) and the viral filtrate was

734 collected. Half of the viral filtrate was autoclaved to eliminate active viruses. The remaining viral

735 filtrate was left unamended. Bacterial communities were resuspended off of the $0.2 \mu \mathrm{m}$ filters in

$7361 \mathrm{~mL}$ autoclaved viral filtrate by shaking for 10 minutes at medium speed on a MoBio Vortex

737 Genie 2. Resuspensions from each filter were pooled to create a single resuspended sample.

738 Resuspended cells were combined 1:120 with either autoclaved (no viruses) or un-autoclaved 
739 (with viruses) viral filtrate. Samples were divided into twelve $20 \mathrm{~mL}$ replicates. Six replicates

740 without viruses and six replicates with viruses were incubated at room temperature. $500 \mu \mathrm{L}$

741 aliquots were taken at the beginning of the incubation and approximately every 24 hours over

742 three days. 16S rRNA and 'Cyano SP'-like RNR gene counts were quantified at each time point

743 by qPCR as described above. 16S amplicon libraries were created as described above for the

744 initial community and the community after 75 hours for all replicates. Growth of individual

745 bacterial populations over the course of the incubation was calculated from qPCR results and

746 their relative abundance. Only bacterial populations that grew in the absence of viruses were

747 retained for further analysis. Susceptibility to viral-mediated mortality was calculated per

748 bacterial population as (Population Abundance Fold-Change Without viruses)/(Population

749 Abundance Fold-Change With Viruses). Higher values indicate greater susceptibility to viral-

750 induced mortality. Growth rates were calculated per $16 \mathrm{~S}$ bacterial population as the fold-change

751 in abundance over time based on relative abundance and qPCR results. Relative growth rates

752 were calculated per $16 \mathrm{~S}$ bacterial population to normalize between replicates. Relative growth

753 rate was defined as the growth rate of a bacterial population relative to the growth rate of the

754 entire community during incubation without viruses.

756 Single-cell genomics

757 Samples from May 18, 2017 were sent to the Bigelow Single Cell Genomics Center (East

758 Boothbay, ME) for sorting of the cyanobacteria and prokaryotic fraction into 384-well plates.

759 Sorting was conducted with the red fluorescence as a function of forward scatter, used to gate for

760 "cyanobacteria", and Syto-9 stained DNA as a function of forward scatter, used to gate for "all

761 prokaryotes". Single cell genome amplification (SAG Generation 2) of sorted cells was 
762 conducted at the Bigelow labs and returned to JHU for marker analysis. RNR and 16S rRNA

763 marker genes were amplified from $2 \mu \mathrm{L}$ of a 1:100 dilution of the amplified genomes according

764 to the protocol above, without the use of emulsions. Positive amplicons were purified and

765 sequenced with the forward primers at the Genetic Resource Core Facility at JHU with an

766 Applied Biosystems 3730xl DNA Analyzer.

767

768 Data availability. Sequences have been deposited in GenBank database under BioProject

769 accession number PRJNA599167.

770 


\section{References}

7721 Suttle, C. A. Marine viruses - major players in the global ecosystem. Nature Reviews

773 Microbiology 5, 801 (2007).

7742 Emerson, J. B. et al. Host-linked soil viral ecology along a permafrost thaw gradient.

$775 \quad$ Nature microbiology 3, 870 (2018).

7763 Reyes, A., Semenkovich, N. P., Whiteson, K., Rohwer, F. \& Gordon, J. I. Going viral: next-generation sequencing applied to phage populations in the human gut. Nature Reviews Microbiology 10, 607 (2012).

7815 Guidi, L. et al. Plankton networks driving carbon export in the oligotrophic ocean.

782

783 Nature 532, 465-470, doi:10.1038/nature16942 (2016).

784 Roux, S. et al. Ecogenomics and potential biogeochemical impacts of globally abundant ocean viruses. Nature 537, 689 (2016). Winget, D. M. et al. Repeating patterns of virioplankton production within an estuarine ecosystem. Proceedings of the National Academy of Sciences 108, 11506-11511 (2011).

8 Chen, X. W. et al. Tide driven microbial dynamics through virus-host interactions in the estuarine ecosystem. Water Res 160, 118-129, doi:10.1016/j.watres.2019.05.051 (2019). Microbiology 5, 801-812, doi:10.1038/nrmicro1750 (2007).

11 Flores, C. O., Valverde, S. \& Weitz, J. S. Multi-scale structure and geographic drivers of

12 Jover, L. F., Cortez, M. H. \& Weitz, J. S. Mechanisms of multi-strain coexistence in

(2013). 65-77 (2013).

Cell 177, 1109-+, doi:10.1016/j.cell.2019.03.040 (2019).

15 Tully, B. J., Graham, E. D. \& Heidelberg, J. F. The reconstruction of 2,631 draft metagenome-assembled genomes from the global oceans. Scientific data 5, 170203 (2018).

6 Burstein, D. et al. Major bacterial lineages are essentially devoid of CRISPR-Cas viral defence systems. Nature communications 7, 10613 (2016).

Hatfull, G. F. Dark matter of the biosphere: the amazing world of bacteriophage diversity. Journal of virology 89, 8107-8110 (2015).

8 Middelboe, M., Chan, A. \& Bertelsen, S. K. in Manual of aquatic viral ecology $118-$ 133 (American Society of Limnology and Oceanography, Inc., 2010).

19 Deng, L. et al. Viral tagging reveals discrete populations in Synechococcus viral genome sequence space. Nature 513, 242-+, doi:10.1038/nature13459 (2014). 
81620 Mosier-Boss, P. A. et al. Use of fluorescently labeled phage in the detection and

817 identification of bacterial species. Appl Spectrosc 57, 1138-1144, doi:Doi 10.1366/00037020360696008 (2003).

21 Allers, E. et al. Single-cell and population level viral infection dynamics revealed by phage FISH, a method to visualize intracellular and free viruses. Environmental microbiology 15, 2306-2318 (2013).

22 Tadmor, A. D., Ottesen, E. A., Leadbetter, J. R. \& Phillips, R. Probing Individual Environmental Bacteria for Viruses by Using Microfluidic Digital PCR. Science 333, 5862, doi:10.1126/science.1200758 (2011). functional genes with phylogenetic markers. The ISME journal 10, 427 (2016).

24 Warwick-Dugdale, J. et al. Long-read viral metagenomics captures abundant and microdiverse viral populations and their niche-defining genomic islands. PeerJ 7, e6800, doi:10.7717/peerj.6800 (2019).

25 Brum, J. R. et al. Patterns and ecological drivers of ocean viral communities. Science 348, 1261498, doi:10.1126/science.1261498 (2015).

26 Gregory, A. C. et al. Genomic differentiation among wild cyanophages despite widespread horizontal gene transfer. BMC Genomics 17, 930, doi:10.1186/s12864-0163286-x (2016).

27 Roux, S. et al. Minimum Information about an Uncultivated Virus Genome (MIUViG). Nat Biotechnol 37, 29-37, doi:10.1038/nbt.4306 (2019).

28 Jang, H. B. et al. Taxonomic assignment of uncultivated prokaryotic virus genomes is enabled by gene-sharing networks. Nature biotechnology 37, 632-639 (2019).

29 Jasna, V., Parvathi, A. \& Dash, A. Genetic and functional diversity of double-stranded DNA viruses in a tropical monsoonal estuary, India. Scientific Reports 8, 16036, doi:10.1038/s41598-018-34332-8 (2018).

30 McDaniel, L. D., Rosario, K., Breitbart, M. \& Paul, J. H. Comparative metagenomics: natural populations of induced prophages demonstrate highly unique, lower diversity viral sequences. Environmental Microbiology 16, 570-585, doi:Doi 10.1111/14622920.12184 (2014).

31 Allen, L. Z. et al. The Baltic Sea Virome: Diversity and Transcriptional Activity of DNA and RNA Viruses. Msystems 2, e00125-00116, doi:10.1128/mSystems.00125-16 (2017).

32 Bench, S. R. et al. Metagenomic characterization of Chesapeake Bay virioplankton. Appl. Environ. Microbiol. 73, 7629-7641 (2007).

33 Kan, J., Evans, S. E., Chen, F. \& Suzuki, M. T. Novel estuarine bacterioplankton in rRNA operon libraries from the Chesapeake Bay. Aquatic Microbial Ecology 51, 55-66 (2008).

34 Chen, F. et al. Diverse and dynamic populations of cyanobacterial podoviruses in the Chesapeake Bay unveiled through DNA polymerase gene sequences. Environmental Microbiology 11, 2884-2892, doi:10.1111/j.1462-2920.2009.02033.x (2009).

35 Kan, J., Suzuki, M. T., Wang, K., Evans, S. E. \& Chen, F. High temporal but low spatial heterogeneity of bacterioplankton in the Chesapeake Bay. Appl. Environ. Microbiol. 73, 6776-6789 (2007).

36 Nasko, D. J. et al. Family A DNA polymerase phylogeny uncovers diversity and replication gene organization in the virioplankton. Frontiers in microbiology 9, 3053 (2018). 
86237 Sakowski, E. G. et al. Ribonucleotide reductases reveal novel viral diversity and predict biological and ecological features of unknown marine viruses. Proceedings of the National Academy of Sciences 111, 15786-15791 (2014).

38 Adriaenssens, E. M. \& Cowan, D. A. Using signature genes as tools to assess environmental viral ecology and diversity. Appl. Environ. Microbiol. 80, 4470-4480 (2014).

39 Harrison, A. O., Moore, R. M., Polson, S. W. \& Wommack, K. E. Reannotation of the ribonucleotide reductase in a cyanophage reveals life history strategies within the virioplankton. Frontiers in microbiology 10, 134 (2019).

40 Martinez-Hernandez, F. et al. Single-virus genomics reveals hidden cosmopolitan and abundant viruses. Nature communications 8, 15892 (2017).

41 Galiez, C., Siebert, M., Enault, F., Vincent, J. \& Soding, J. WIsH: who is the host? Predicting prokaryotic hosts from metagenomic phage contigs. Bioinformatics 33, 31133114, doi:10.1093/bioinformatics/btx383 (2017).

42 Galiez, C., Siebert, M., Enault, F., Vincent, J. \& Söding, J. WIsH: who is the host? Predicting prokaryotic hosts from metagenomic phage contigs. Bioinformatics 33, 31133114 (2017).

43 Kavagutti, V. S., Andrei, A. S., Mehrshad, M., Salcher, M. M. \& Ghai, R. Phage-centric ecological interactions in aquatic ecosystems revealed through ultra-deep metagenomics. Microbiome 7, 135, doi:10.1186/s40168-019-0752-0 (2019).

44 Holmfeldt, K., Middelboe, M., Nybroe, O. \& Riemann, L. Large variabilities in host strain susceptibility and phage host range govern interactions between lytic marine

45 Tzortziou, M. et al. Tidal marshes as a source of optically and chemically distinctive colored dissolved organic matter in the Chesapeake Bay. Limnology and Oceanography 53, 148-159, doi:DOI 10.4319/lo.2008.53.1.0148 (2008).

46 Chai, T. J. Characteristics of Escherichia-Coli Grown in Bay Water as Compared with Rich Medium. Applied and Environmental Microbiology 45, 1316-1323, doi:Doi 10.1128/Aem.45.4.1316-1323.1983 (1983).

47 Martiny, J. B., Riemann, L., Marston, M. F. \& Middelboe, M. Antagonistic coevolution of marine planktonic viruses and their hosts. (2014).

48 Sieradzki, E. T., Ignacio-Espinoza, J. C., Needham, D. M., Fichot, E. B. \& Fuhrman, J. A. Dynamic marine viral infections and major contribution to photosynthetic processes shown by spatiotemporal picoplankton metatranscriptomes. Nat Commun 10, 1169, doi:10.1038/s41467-019-09106-z (2019).

49 Moniruzzaman, M. et al. Virus-host relationships of marine single-celled eukaryotes resolved from metatranscriptomics. Nat Commun 8, 16054, doi:10.1038/ncomms16054 (2017).

50 Duffy, S., Turner, P. E. \& Burch, C. L. Pleiotropic costs of niche expansion in the RNA bacteriophage 66. Genetics 172, $751-757$ (2006).

51 Sunagawa, S. et al. Structure and function of the global ocean microbiome. Science 348, 1261359, doi:10.1126/science.1261359 (2015).

52 Roux, S. et al. Ecogenomics and potential biogeochemical impacts of globally abundant ocean viruses. Nature 537, 689-+, doi:10.1038/nature19366 (2016).

53 Deng, L. et al. Contrasting life strategies of viruses that infect photo-and heterotrophic bacteria, as revealed by viral tagging. MBio 3, e00373-00312 (2012). 
$90854 \quad$ Labonté, J. M. et al. Single-cell genomics-based analysis of virus-host interactions in

909 marine surface bacterioplankton. The ISME journal 9, 2386-2399 (2015).

$91055 \quad$ Adriaenssens, E. M. \& Cowan, D. A. Using Signature Genes as Tools To Assess Environmental Viral Ecology and Diversity. Applied and Environmental Microbiology 80, 4470-4480, doi:10.1128/Aem.00878-14 (2014).

56 Martinez-Hernandez, F. et al. Single-virus genomics reveals hidden cosmopolitan and abundant viruses. Nat Commun 8, 15892, doi:10.1038/ncomms15892 (2017).

916

57 Martinez-Hernandez, F. et al. Droplet Digital PCR for Estimating Absolute Abundances of Widespread Pelagibacter Viruses. Front Microbiol 10, 1226, doi:10.3389/fmicb.2019.01226 (2019).

58 Vik, D. R. et al. Putative archaeal viruses from the mesopelagic ocean. PeerJ 5, e3428, doi:10.7717/peerj.3428 (2017).

59 Jover, L. F., Romberg, J. \& Weitz, J. S. Inferring phage-bacteria infection networks from time-series data. Royal Society Open Science 3, 160654 (2016). Labonte, J. M. et al. Single-cell genomics-based analysis of virus-host interactions in marine surface bacterioplankton. Isme J 9, 2386-2399, doi:10.1038/ismej.2015.48 (2015).

61 Brankatschk, R., Bodenhausen, N., Zeyer, J. \& Burgmann, H. Simple Absolute Quantification Method Correcting for Quantitative PCR Efficiency Variations for Microbial Community Samples. Applied and Environmental Microbiology 78, 44814489, doi:10.1128/Aem.07878-11 (2012).

62 Baran, N., Goldin, S., Maidanik, I. \& Lindell, D. Quantification of diverse virus populations in the environment using the polony method. Nature Microbiology $\mathbf{3}$, doi:10.1038/s41564-017-0045-y (2018).

932

63 Russell, D. A. \& Hatfull, G. F. PhagesDB: the actinobacteriophage database.

933 Bioinformatics 33, 784-786, doi:10.1093/bioinformatics/btw711 (2017). natans, Escherichia coli, and Pseudomonas aeruginosa. Applied and Environmental Microbiology 64, 575-580 (1998).

65 Peters, D. L., Lynch, K. H., Stothard, P. \& Dennis, J. J. The isolation and characterization of two Stenotrophomonas maltophilia bacteriophages capable of cross-taxonomic order infectivity. BMC Genomics 16, 664, doi:10.1186/s12864-015-1848-y (2015).

66 Paez-Espino, D. et al. Uncovering Earth's virome. Nature 536, 425-+, doi:10.1038/nature19094 (2016).

67 Chiura, H. X. Generalized gene transfer by virus-like particles from marine bacteria. Aquatic Microbial Ecology 13, 75-83 (1997). Hanski, I., Hansson, L. \& Henttonen, H. SPECIALIST PREDATORS, GENERALIST PREDATORS, AND THE MICROTINE RODENT CYCLE. Journal of Animal Ecology 60, 353-367, doi:10.2307/5465 (1991).

69 Johnke, J. et al. A Generalist Protist Predator Enables Coexistence in Multitrophic Predator-Prey Systems Containing a Phage and the Bacterial Predator Bdellovibrio. Front Ecol Evol 5 (2017).

70 John, S. G. et al. A simple and efficient method for concentration of ocean viruses by chemical flocculation. Environmental microbiology reports 3, 195-202 (2011).

71 Bolger, A. M., Lohse, M. \& Usadel, B. Trimmomatic: a flexible trimmer for Illumina sequence data. Bioinformatics 30, 2114-2120 (2014). 
95372 Bankevich, A. et al. SPAdes: A New Genome Assembly Algorithm and Its Applications

954

955

956

957

958

959

960

961

962

963

964

965

966

967

968

969

970

971

972

973

974

975

976

977

978

979

980

981

982

983

984

985

986

987

988

989

990

991

992

993

994

995

996

997

998

to Single-Cell Sequencing. Journal of Computational Biology 19, 455-477,

doi:10.1089/cmb.2012.0021 (2012).

73 Uritskiy, G. V., DiRuggiero, J. \& Taylor, J. MetaWRAP — a flexible pipeline for genomeresolved metagenomic data analysis. Microbiome 6, 158 (2018).

74 Kang, D. D., Froula, J., Egan, R. \& Wang, Z. MetaBAT, an efficient tool for accurately reconstructing single genomes from complex microbial communities. PeerJ 3, e1 165 (2015).

75 Wu, Y.-W., Simmons, B. A. \& Singer, S. W. MaxBin 2.0: an automated binning algorithm to recover genomes from multiple metagenomic datasets. Bioinformatics 32, 605-607 (2015).

76 Song, W.-Z.\& Thomas, T. Binning_refiner: improving genome bins through the combination of different binning programs. Bioinformatics 33, 1873-1875 (2017).

77 Parks, D. H., Imelfort, M., Skennerton, C. T., Hugenholtz, P. \& Tyson, G. W. CheckM: assessing the quality of microbial genomes recovered from isolates, single cells, and metagenomes. Genome research 25, 1043-1055 (2015).

78 Menzel, P., Ng, K. L. \& Krogh, A. Fast and sensitive taxonomic classification for metagenomics with Kaiju. Nat Commun 7, 11257, doi:10.1038/ncomms11257 (2016).

79 Schütze, T. et al. A streamlined protocol for emulsion polymerase chain reaction and subsequent purification. Analytical biochemistry 410, 155-157 (2011).

80 Bolyen, E. et al. QIIME 2: Reproducible, interactive, scalable, and extensible microbiome data science. Report No. 2167-9843, (PeerJ Preprints, 2018).

81 DeSantis, T. Z. et al. Greengenes, a chimera-checked 16S rRNA gene database and workbench compatible with ARB. Appl. Environ. Microbiol. 72, 5069-5072 (2006).

82 Hurwitz, B. L., Deng, L., Poulos, B. T. \& Sullivan, M. B. Evaluation of methods to concentrate and purify ocean virus communities through comparative, replicated metagenomics. Environmental Microbiology 15, 1428-1440 (2013).

83 Bushnell, B. BBMap: A Fast, Accurate, Splice-Aware Aligner,

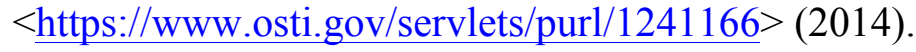

84 De Coster, W., D'Hert, S., Schultz, D. T., Cruts, M. \& Van Broeckhoven, C. NanoPack: visualizing and processing long-read sequencing data. Bioinformatics 34, 2666-2669, doi:10.1093/bioinformatics/bty149 (2018).

85 Kolmogorov, M., Yuan, J., Lin, Y. \& Pevzner, P. A. Assembly of long, error-prone reads using repeat graphs. Nat Biotechnol 37, 540-+, doi:10.1038/s41587-019-0072-8 (2019).

86 Walker, B. J. et al. Pilon: An Integrated Tool for Comprehensive Microbial Variant Detection and Genome Assembly Improvement. Plos One 9, e112963, doi:10.1371/journal.pone.0112963 (2014).

87 Li, H. \& Durbin, R. Fast and accurate short read alignment with Burrows-Wheeler transform. Bioinformatics 25, 1754-1760, doi:10.1093/bioinformatics/btp324 (2009).

88 Roux, S., Enault, F., Hurwitz, B. L. \& Sullivan, M. B. VirSorter: mining viral signal from microbial genomic data. PeerJ 3, e985, doi:10.7717/peerj.985 (2015).

89 Ren, J. et al. Identifying viruses from metagenomic data using deep learning. Quantitative Biology, doi:10.1007/s40484-019-0187-4 (2020).

90 Bolduc, B., Youens-Clark, K., Roux, S., Hurwitz, B. L. \& Sullivan, M. B. iVirus: facilitating new insights in viral ecology with software and community data sets imbedded in a cyberinfrastructure. Isme J 11, 7-14, doi:10.1038/ismej.2016.89 (2017). 
$99991 \quad$ Langmead, B. \& Salzberg, S. L. Fast gapped-read alignment with Bowtie 2. Nat Methods 9, 357-U354, doi:10.1038/Nmeth.1923 (2012).

1001

92 Jang, H. B. et al. Taxonomic assignment of uncultivated prokaryotic virus genomes is

1002

1003

1004 enabled by gene-sharing networks. Nat Biotechnol 37, 632-+, doi:10.1038/s41587-0190100-8 (2019).

1005

1006

1007

1008

1009

93 Hyatt, D. et al. Prodigal: prokaryotic gene recognition and translation initiation site identification. BMC Bioinformatics 11, 119, doi:10.1186/1471-2105-11-119 (2010).

94 Smoot, M. E., Ono, K., Ruscheinski, J., Wang, P. L. \& Ideker, T. Cytoscape 2.8: new features for data integration and network visualization. Bioinformatics 27, 431-432, doi:10.1093/bioinformatics/btq675 (2011).

1010

95 Caporaso, J. G. et al. QIIME allows analysis of high-throughput community sequencing

101196 Warnes, G. R. et al. gplots: Various R programming tools for plotting data. (2015).

101297 Langmead, B. \& Salzberg, S. L. Fast gapped-read alignment with Bowtie 2. Nature

1013 methods 9, 357 (2012).

101498 Zhu, W., Lomsadze, A. \& Borodovsky, M. Ab initio gene identification in metagenomic

1015

1016 sequences. Nucleic acids research 38, e132-e132 (2010).

1017

1018

1019

99 Suzek, B. E., Huang, H., McGarvey, P., Mazumder, R. \& Wu, C. H. UniRef: comprehensive and non-redundant UniProt reference clusters. Bioinformatics 23, 12821288 (2007).

1020

1021

1022

1023

1024

100 Katoh, K. \& Standley, D. M. MAFFT multiple sequence alignment software version 7: improvements in performance and usability. Molecular biology and evolution 30, 772780 (2013).

101 Kearse, M. et al. Geneious Basic: an integrated and extendable desktop software platform for the organization and analysis of sequence data. Bioinformatics 28, 1647-1649 (2012).

102 Martin, M. Cutadapt removes adapter sequences from high-throughput sequencing reads. EMBnet. journal 17, 10-12 (2011).

1026

103 Fu, L., Niu, B., Zhu, Z., Wu, S. \& Li, W. CD-HIT: accelerated for clustering the nextgeneration sequencing data. Bioinformatics 28, 3150-3152 (2012).

1027

1028

104 Dereeper, A. et al. Phylogeny. fr: robust phylogenetic analysis for the non-specialist. Nucleic acids research 36, W465-W469 (2008).

1030

1031

\section{Acknowledgements}

The authors would like to thank the Smithsonian Environmental Research Center and

1034 Katrine Lohan for providing access to their facilities during sample collection. This work was

1035 supported by the National Science Foundation Biological Oceanography (awards \#1820652,

$1036 \quad \# 1829831$ and \#1756314) and a Gordon and Betty Moore Foundation Investigator Award

1037 (\#3790). Part of this project was conducted using computational resources at the Maryland 
1038 Advanced Research Computing Center (MARCC) and the Ohio Supercomputer Center (OSC)

1039 for High Performance Computing.

\section{Author Contributions}

1042 EGS and SPP conceived of the work. EGS conducted all field-work, epicPCR analysis, and

1043 incubation experiments associated with this work. EGS, KAW, and SPP conducted the

1044 experimental and computational analysis for the bacterial metagenome and 16S rRNA gene

1045 libraries. EGS, FT, AAZ, and OZ conducted experimental and computational analysis for the

1046 viral metagenomic libraries. EGS, KAW, and SPP conducted the bioinformatic host prediction.

1047 EGS wrote the manuscript. EGS, AAZ, OZ, MBS, and SPP edited the manuscript.

1049 Competing interests

1050 The authors declare no competing financial interests.

1051

1052 Additional information

1053 Supplementary information is available for this paper.

1054 Correspondence and requests for materials should be addressed to EGS and SPP. 